Research Article: Methods/New Tools / Novel Tools and Methods

\title{
Targeting neurons with functional oxytocin receptors: A novel set of simple knock-in mouse lines for oxytocin receptor visualization and manipulation
}

https://doi.org/10.1523/ENEURO.0423-21.2022

Cite as: eNeuro 2022; 10.1523/ENEURO.0423-21.2022

Received: 6 October 2021

Revised: 13 January 2022

Accepted: 14 January 2022

This Early Release article has been peer-reviewed and accepted, but has not been through the composition and copyediting processes. The final version may differ slightly in style or formatting and will contain links to any extended data.

Alerts: Sign up at www.eneuro.org/alerts to receive customized email alerts when the fully formatted version of this article is published. 4.0 International license, which permits unrestricted use, distribution and reproduction in any medium provided that the original work is properly attributed. 
Manuscript Title Page

\section{Manuscript title}

Targeting neurons with functional oxytocin receptors:

A novel set of simple knock-in mouse lines for oxytocin receptor visualization and manipulation

\section{Abbreviated title}

Targeting neurons with functional oxytocin receptors

\section{List of all authors names and affiliations}

Yukiko U. Inoue ${ }^{1}$, Hideki Miwa ${ }^{2}$, Kei Hori ${ }^{1}$, Ryosuke Kaneko ${ }^{3}$, Yuki Morimoto ${ }^{1}$, Eriko Koike ${ }^{1}$, Junko Asami ${ }^{1}$, Satoshi Kamijo ${ }^{2}$, Mitsuhiko Yamada ${ }^{2}$, Mikio Hoshino ${ }^{1}$, Takayoshi Inoue ${ }^{1}$

${ }^{1}$ Department of Biochemistry and Cellular Biology, National Institute of Neuroscience, National Center of Neurology and Psychiatry, Kodaira, Tokyo 187-8502, Japan

2 Department of Neuropsychopharmacology, National Institute of Mental Health, National Center of Neurology and Psychiatry, Kodaira, Tokyo 187-8553, Japan

${ }^{3}$ KOKORO-Biology Group, Laboratories for Integrated Biology, Graduate School of Frontier Biosciences, Osaka University, Suita, Osaka 565-0871, Japan

\section{Author contributions}

Y.U.I., H.M., and R.K. designed the experiments. Y.U.I., H.M., K.H., R.K., Y.M., E.K., J.A. and S.K. performed the experiments. Y.U.I., H.M., K.H., R.K., M.Y., M.H., and T.I. analyzed and discussed the results. Y.U.I., H.M., and T.I. wrote the manuscript. All authors have read and agreed to the final version of the manuscript.

5. Correspondence should be addressed to Yukiko U. Inoue (yinn3@ncnp.go.jp) and Takayoshi Inoue (tinoue@ncnp.go.jp)

\section{Number of Figures, 5}

7. Number of Tables, 0

8. Number of Multimedia, 2

9. Number of words for Abstract, 266

10. Number of words for Significance Statement, 124

11. Number of words for Introduction, 840

12. Number of words for Discussion, 1,218

\section{Acknowledgements}

This work was supported by JSPS KAKENHI Grant Numbers 16K10004, 17H05967, 19H04922, $20 K 06467$ to Y.U.I, 18KK0442, 19 K08033 to H.M., 17H05937, 19H04895, $20 \mathrm{H02932}$ to R.K.. This work is also supported by Intramural Research Grants for Neurological and Psychiatric Disorders of NCNP (1-1, 30-9, 3-9) to H.M., M.Y., M.H. and T.I., and Japan Agency for Medical Research and Development (AMED) Grand Number JP21wm0425005 to M.H., 21 ek0109490h0002 to T.I.. Viral vectors used in this work were provided by the program for Brain Mapping by Integrated Neurotechnologies for Disease Studies (Brain/MINDS) from AMED under the Grant Number JP20dm0207057 and JP21dm0207111. The authors thank all the lab members in Department of Biochemistry and Cellular Biology at NCNP for their supports.

\section{Conflict of interest}


50 Authors report no conflict of interest.

51

52

53

54 16. Material Availability

55 Four knock-in mouse lines generated in this study will be made available with a materials transfer 56 agreement.

57

58

59

60

61

62

63

64

65

66

67

68

69

70

71

72

73

74

75

76

77

78

79

80

81

82

83

84

85

86 
87 Targeting neurons with functional oxytocin receptors:

88 A novel set of simple knock-in mouse lines for oxytocin receptor visualization and

89 manipulation

90

91

92

Abstract

93 The neuropeptide oxytocin (Oxt) plays important roles in modulating social behaviors.

94 Oxytocin receptor (Oxtr) is abundantly expressed in the brain and its relationship to

95 socio-behavioral controls has been extensively studied using mouse brains. Several genetic

96 tools to visualize and/or manipulate Oxtr-expressing cells, such as fluorescent reporters and

97 Cre recombinase drivers, have been generated by ES-cell based gene targeting or bacterial

98 artificial chromosome (BAC) transgenesis. However, these mouse lines displayed some

99 differences in their Oxtr expression profiles probably due to the complex context and

100 integrity of their genomic configurations in each line. Here we apply our sophisticated

101

102

103

104 genome-editing techniques to the Oxtr locus, systematically generating a series of knock-in mouse lines, in which its endogenous transcriptional regulations are intactly preserved and evaluate their expression profiles to ensure the reliability of our new tools. We employ the epitope tagging strategy, with which C-terminally fused tags can be detected by highly specific antibodies, to successfully visualize the Oxtr protein distribution on the neural membrane with super-resolution imaging for the first time. By utilizing T2A self-cleaving peptide sequences, we also induce proper expressions of tdTomato reporter, codon-improved Cre recombinase, and spatiotemporally inducible Cre-ERT2 in Oxtr-expressing neurons. Electrophysiological recordings from tdTomato-positive cells in the reporter mice support the validity of our tool design. Retro-orbital injections of AAV-PHP.eB vector into the Cre line further enabled visualization of recombinase activities in the appropriate brain regions. Moreover, the first-time Cre-ERT2 line drives Cre-mediated recombination in a spatiotemporally controlled manner upon tamoxifen administration. These tools thus provide an excellent resource for future functional studies in Oxt-responsive neurons and should prove of broad interest in the field.

\section{Significance Statements}

119 Here we develop a novel series of genome-edited mouse lines to help understand the circuit

120 mechanisms underlying Oxt actions, by enabling the visualization and manipulation of

121 Oxtr-expressing neurons in a manner precisely reflecting their endogenous expression 
profiles. The epitope tagging strategy allows super-resolution imaging to decipher the 3-D distributions of Oxtr protein on neural membranes for the first time. The red fluorescent tdTomato reporter serves as a reliable visualization tool for Oxtr-expressing cells, whose expression profiles are validated using electrophysiological recordings. Both iCre and iCre-ERT2 drivers, which properly induce recombinase activities in Oxtr-expressing cells, would largely contribute to viral vector-dependent functional analyses and fate mapping studies. These tools thus offer a new option for the research communities to improve our knowledge of Oxt-Oxtr circuitry.

\section{Introduction}

The neuropeptide oxytocin (Oxt) has attracted great attention not only from researchers but also from the general public because of its profound social (Froemke and Young, 2021), pro-social (Marsh et al., 2020), anxiolytic (Neumann and Slattery, 2016), and anti-stress (Onaka and Takayanagi, 2019) behavioral effects in animals. Thanks to the advanced neuro-technologies, including optogenetics (Deisseroth, 2011; Grinevich and Neumann, 2021), chemogenetics (Grinevich and Neumann, 2021; Grund et al., 2019), and calcium imaging (Hung et al., 2017; Maldonado et al., 2021; Resendez et al., 2020), the complex neurobiology of the Oxt system has been gradually revealed over the last decade. The oxytocin receptor (Oxtr), a member of the G-protein-coupled receptor (GPCR) family, processes and transfers signals into the cytoplasm to modulate downstream circuitries (Grinevich and Neumann, 2021). Although a growing number of studies have shed light on Oxt-Oxtr signaling in various brain regions such as the prefrontal cortex (PFC) (Nakajima et al., 2014; Tan et al., 2019), lateral septum (Horiai et al., 2020; Menon et al., 2018), nucleus accumbens (Dölen et al., 2013; Nardou et al., 2019), hippocampus (Owen et al., 2013; Raam et al., 2017), ventral tegmental area (Hung et al., 2017), and others, each study has used different tools to visualize and manipulate Oxtr-expressing neurons.

Some studies have used an antibody (called OXTR-2) generated in a research laboratory to detect Oxtr proteins (Marlin et al., 2015; Mitre et al., 2016). Unfortunately, this antibody is not available for the public to replicate the experimental findings. In addition, because of the general difficulty in generating antibodies to lipophilic GPCRs with low epitope exposures, no anti-Oxtr antibody suitable for immunohistochemistry is commercially available. From these reasons, the Oxtr-Venus knock-in mouse line was generated (Yoshida et al., 2009) and used for receptor visualization (Dölen et al., 2013; Hung et al., 2017; Newmaster et al., 2020). However, contrary to our expectations, expressions of the Venus 
reporter in the brain was reported to largely decrease during development (Newmaster et al., 2020). In this reporter line, the 1st coding exon had been replaced with Venus sequences (Extended Data Fig.1-1b). The resulting knock-in allele lacks its endogenous configurations of intron and 3' UTR sequences, both of which likely contain transcriptional regulatory elements essential for the spatiotemporally coordinated Oxtr expressions. This might considerably affect the Venus reporter expression profiles.

Cre recombinase driver mice also play pivotal roles in both conditional knockout experiments and viral vector-dependent functional analyses. Although two Oxtr-Cre knock-in lines utilizing internal ribosome entry site (IRES) sequences for their bicistronic expressions have been generated (Hidema et al., 2016; Ryan et al., 2017) (Extended Data Fig.1-1c, 1-1d), those expression profiles reported were relatively sparse and did not well overlap with each other, probably due to the different context and integrity of their genomic configurations. In addition, the Oxtr-T2A-Cre-D line recently became available from The Jackson Laboratory

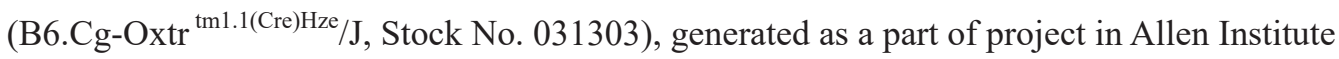
for Brain Science (Daigle et al., 2018) (Extended Data Fig.1-1e), yet its detailed expression profile has not been described.

Endogenously, the Oxtr gene locus consists of two coding exons divided by a long intron (Extended Data Fig.1-1a). In prairie vole and human, some single-nucleotide polymorphisms (SNPs) in this long intron have been reported to be associated with Oxtr expression densities or social behavioral phenotypes (Jurek and Neumann, 2018; King et al., 2016). The authors suggested that these SNPs might overlap with cis-regulatory elements in the intron (King et al., 2016). The 3' UTR of the Oxtr gene has also been reported to contain nucleotide variations that are related to social behavioral phenotypes in humans (Jurek and Neumann, 2018). Considering these reports, it must be critical to design knock-in lines by maximally preserving the original transcriptional regulations, so as to precisely recapitulate endogenous Oxtr-expression profiles.

For proteins with no antibodies available for immunohistological detection, the epitope tagging strategy could be a promising breakthrough (Brizzard, 2008). Although the previously reported Oxtr-IRES-Cre line was designed to harbor an HA-tag at the C-terminus of the receptor protein (Extended Data Fig.1-1c), the epitope tag could not be detected in the brain tissues probably because of its relatively lower expression levels, caused by the non-native exon/intron configuration (Hidema et al., 2016). In this study, we first employed the novel PA-tag system in which human podoplanin-derived epitope tag is recognized by its highly specific monoclonal antibody (Fujii et al., 2014). Since this system has been successfully applied to many in vitro procedures, such as protein purification and 
visualization, we anticipated that it would also work in vivo to determine the subcellular distributions of endogenous Oxtr protein in neurons. We next utilized the HA-tag system, because its commercially available antibody (Cell Signaling Technology, C29F4, \#3724) has been successfully adopted by in vivo genome editing technique, SLENDR, to clearly map the subcellular localization of endogenous proteins in brain tissues with low background staining (Mikuni et al., 2016).

To achieve these concepts, here we apply our refined genome-editing skills to the Oxtr locus to generate four novel knock-in mouse lines, and evaluate their expression profiles to ensure the reliability in vivo.

\section{Materials and Methods}

\section{Animals}

All animal procedures were performed in accordance with the Animal Care Committee's regulations. B6C3F1 female mice for fertilized eggs collection and ICR female mice as surrogate mothers were purchased from SLC Japan. One-cell stage zygotes were obtained by mating B6C3F1 stud males with super-ovulated females. Pronuclear injections and mouse transgenesis experiments were performed by standard protocols (Nagy et al., 2003). C57BL/6J mice for back-crossing were purchased from Charles River Japan.

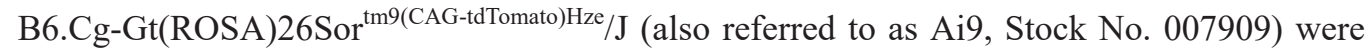
introduced from The Jackson Laboratory.

\section{Preparation of CRISPR components}

To insert epitope-tag, reporter, and recombinase sequences into the Oxtr locus via the CRISPR/Cas9 system, the guide RNA sequences closest to the stop codon TGA in the $2^{\text {nd }}$ exon (depicted in Fig.1a) were selected. Two parts of the CRISPR guide RNA, crRNA and tracrRNA, were chemically synthesized (FASMAC, Japan) and recombinant Cas9 protein (EnGen Cas9 NLS S. pyogenes) was purchased from New England Biolabs. For pronuclear injections, equimolar crRNA and tracrRNA were mixed and annealed $\left(94^{\circ} \mathrm{C}, 2 \mathrm{~min}\right.$, then at room temperature for $10 \mathrm{~min}$ ).

\section{Generation of the $O x t r^{1 \times P A-t d T o m}$ mouse line}

To insert $1 \times$ PA-tag, T2A self-cleaving peptide, and tdTomato sequences just upstream from the stop codon (Fig. 1b), the targeting vector containing those sequences flanked by $2.9 \mathrm{~kb}$ homology arms on both sides was constructed by the In-Fusion cloning system (Takara Bio). 
227 The mixture of annealed crRNA/tracrRNA, Cas9 protein, and the targeting vector was

228 injected into mouse fertilized eggs at the concentrations of 38,50 , and $25 \mathrm{ng} / \mu \mathrm{l}$, and the eggs

229 were transferred into the oviduct of surrogate mothers to raise pups.

230

231 Generation of the Oxtr $^{3 \times P A}$ mouse line

232 To insert three tandem copies of PA-tag sequences just upstream from the stop codon (Fig.1b),

$2333 \times$ PA-tag sequences flanked by 129 and 118 bp homology arms were artificially synthesized

234 (Integrated DNA Technologies). To avoid unwanted excision of PA-tag sequences by the

235 internal recombination among three copies, synonymous substitutions were introduced into

236 each tag sequences. By using this synthesized DNA as the template, long single-stranded

237 DNA (ssDNA) donors were prepared with the Phospho-PCR method (Guide-it Long ssDNA

238 Production System, Takara Bio) (Inoue et al., 2021). The mixture of annealed

239 crRNA/tracrRNA, Cas9 protein, and long ssDNA donor was injected into mouse zygotes at

240 the concentrations of 38,50 , and $25 \mathrm{ng} / \mu \mathrm{l}$, and the zygotes were transferred into the oviduct

241 of surrogate mothers to raise pups.

\section{Generation of the Oxtr $^{1 \times P A-i C r e}$ mouse line}

245

To insert $1 \times$ PA-tag sequences, T2A self-cleaving peptide sequences, and codon-improved Cre recombinase (iCre) (Shimshek et al., 2002) sequences just upstream from the stop codon (Fig.1b), the targeting vector containing those sequences flanked by 245 and 294 bp homology arms was constructed via the In-Fusion cloning system. By using this vector as the template, long ssDNA donors were synthesized by the Phospho-PCR method. The mixture of annealed crRNA/tracrRNA, Cas9 protein, and long ssDNA donor was injected into mouse zygotes at the concentrations of 38,50 , and $18.5 \mathrm{ng} / \mu \mathrm{l}$, and the zygotes were transferred into the oviduct of surrogate mothers to raise pups.

\section{Generation of the Oxtr $^{3 \times H A-i C r e E R T 2}$ mouse line}

254 To insert three copies of HA-tag, T2A self-cleaving peptide, iCre, and mutated ligand binding 255 domain of the human estrogen receptor (ERT2) sequences (Indra et al., 1999) just upstream 256 from the stop codon (Fig. 1b), the targeting vector containing those sequences flanked by $2 \mathrm{~kb}$ homology arms on both sides was constructed by combining artificial gene synthesis

258 (Integrated DNA technologies) and In-Fusion cloning system. Synonymous base substitutions were introduced into each HA-tag sequence to obviate unwanted internal recombination among them. The mixture of crRNA/tracrRNA complex, Cas9 protein, and the targeting 
261

262

263

264

265

266

267

268

269

270

271

272

273

274

275

276

277

278

279

280

281

282

283

284

285

286

287

288

289

290

291

292

293

294

295

vector was injected into mouse 1-cell embryos at the concentrations of 50,100, and $15 \mathrm{ng} / \mu \mathrm{l}$, and the embryos were transferred into the oviduct of surrogate mothers to raise pups.

\section{Validation of the founder knock-in mice and their backcrossing}

Genomic DNAs were prepared from newborns' tail by proteinase K treatment in Lysis Buffer (Takara Bio). Knock-in mice were screened by PCR using Tks Gflex DNA polymerase (Takara Bio) or PrimeSTAR GXL DNA polymerase (Takara Bio). To exclude the possibilities that the donor DNAs had been randomly integrated into the genome, a subset of genotyping primers was designed external to the donor DNAs' homology arms. For knock-in candidates, PCR products were analyzed by Sanger sequencing to confirm carrying correct insertions. Obtained founders were backcrossed with $\mathrm{C} 57 \mathrm{BL} / 6 \mathrm{~J}$ to get $\mathrm{F} 1$ heterozygous progenies, which were again sequence-verified to harbor the designed knock-in allele. F1 heterozygous mice were then intercrossed to generate homozygous knock-in mice. For histological, electrophysiological, and viral analyses, homozygous knock-in mice after F3 generations were used.

\section{Antibodies and viral vectors}

Primary antibodies and dilutions used were Guinea Pig anti-tdTomato (1:2000, Frontier Institute AB_2631185), Rat anti-PA-tag (1:2000, FUJIFILM Wako Chemicals NZ-1 012-25861), Rabbit anti-HA-tag (1:2000, Cell Signaling Technology C29F4 3724S), Goat anti-Somatostatin (1:2000, Santa Cruz Biotechnology sc-7819), Guinea Pig anti-GFP (1:1000, Frontier Institute AB_2571575), Rabbit anti-Cux1 (CDP, 1:2000, Santa Cruz Biotechnology sc-13024), and Rabbit anti-Oxytocin (1:2000, IMMUNOSTAR 20068). Appropriate Alexa Fluor-conjugated secondary antibodies were from Thermo Fisher Scientific, abcam, or Jackson ImmunoResearch and used at dilutions of 1:600.

AAV-PHP.eB CBh_FLEX-GFP WPRE was kindly gifted by Dr. Hirokazu Hirai (the program for Brain Mapping by Integrated Neurotechnologies for Disease Studies (Brain/MINDS), AMED).

\section{Immunohistochemistry}

Brain samples were prepared from 2- to 11-week-old male homozygous knock-in mice and immunohistochemically analyzed by standard protocols. Briefly, brains were intracardially perfused with $4 \%$ paraformaldehyde (PFA) in phosphate-buffered saline (PBS; pH 7.4), post-fixed with the same fixative for $16 \mathrm{hr}$, and cryo-protected with $30 \%$ sucrose in PBS, then embedded in OCT compound (Sakura Finetek Japan) to be frozen. Brains were sliced into 40 
$\mu \mathrm{m}$ coronal sections using a Leica cryostat. Sections were treated in blocking buffer $(10 \%$ normal donkey serum, $0.3 \%$ Triton X-100 in PBS) for $1 \mathrm{hr}$ at room temperature with gentle agitation. Sections were then incubated with primary antibodies in blocking solution (3\% normal donkey serum, $0.3 \%$ Triton X-100 in PBS) overnight at $4{ }^{\circ} \mathrm{C}$. After being washed with PBS, sections were treated with appropriate Alexa Fluor-conjugated secondary antibodies (Thermo Fisher Scientific, abcam, or Jackson ImmunoResearch) and DAPI for $1 \mathrm{hr}$ at room temperature. After washing, sections were mounted onto glass slides with Permafluor (Thermo Fisher scientific) or VECTASHIELD Vibrance antifade mounting medium (Vector Laboratories). Fluorescent images were obtained on a KEYENCE BZ-X710. For confocal and super-resolution imaging, an OLYMPUS SpinSR10 was used to take z-stacked images. Movies were constructed by using an OLYMPUS cellSens software.

\section{Electrophysiological recordings from tdTomato-positive neurons in the $O x t{ }^{1 \times P A-t d T o m}$} line

Coronal prefrontal cortex slices (300 $\mu \mathrm{m}$ thickness) were prepared from 4- to 6-week-old male homozygous Oxtr ${ }^{1 \times P A-t d T o m}$ mice, using standard procedures (Miwa et al., 2008). Slices were perfused with artificial cerebrospinal fluid (ACSF) that was saturated with $95 \% \mathrm{O}_{2}$ and $5 \% \mathrm{CO}_{2}$ at $30 \pm 1^{\circ} \mathrm{C}$ and contained (in $\mathrm{mM}$ ) $119 \mathrm{NaCl}, 3.5 \mathrm{KCl}, 2.5 \mathrm{CaCl}_{2}, 1.3 \mathrm{MgSO}_{4}, 1.0$ $\mathrm{NaH}_{2} \mathrm{PO}_{4}, 26.2 \mathrm{NaHCO}_{3}$, and $11 \mathrm{D}$-glucose. Spontaneous spiking in tdTomato-expressing neurons in layer II and $\mathrm{V}$ of the prefrontal cortex (PFC) was acquired in voltage clamp cell-attached configuration with a MultiClamp 700B patch-clamp amplifier (Molecular Devices, Union City, CA, USA). The pipette solution contained ACSF, and the patch was held at $0 \mathrm{mV}$. Baseline firing was acquired for $5 \mathrm{~min}$, followed by bath application of $1 \mu \mathrm{M}\left[\mathrm{Thr}^{4}\right.$, $\left.\mathrm{Gly}^{7}\right]$-oxytocin (TGOT) or $1 \mu \mathrm{M}\left(\mathrm{d}\left(\mathrm{CH}_{2}\right)_{5}{ }^{1}, \mathrm{Tyr}(\mathrm{Me})^{2}, \mathrm{Thr}^{4}, \mathrm{Orn}^{8}\right.$, des-Gly-NH$\left.{ }_{2}{ }^{9}\right)$-vasotocin (OTA) for $10 \mathrm{~min}$. The signal was filtered at $1 \mathrm{kHz}$ and digitized at $20 \mathrm{kHz}$ with National Instruments DAQ board (USB-6341) and Igor Pro (Wavemetrics) with the NeuroMatic software package (Rothman and Silver, 2018). TGOT and OTA were obtained from Bachem (Torrance, CA).

\section{Intravenous injection of AAV-PHP.eB vector to the $O x t{ }^{1 \times P A-i C r e}$ line}

After anesthesia with isoflurane (4\%), $100 \mu \mathrm{l}$ of viral solution (AAV-PHP.eB CBh_FLEX-GFP WPRE; $2.5 \times 10^{12} \mathrm{vg} / \mathrm{ml}$ ) was intravenously injected into the retro-orbital sinus of Oxtr ${ }_{1 \times P A-i C r e /+}$ mice using a $1.0 \mathrm{ml}$ syringe with a 27 -gauge needle. Four weeks after the injection, the mice were anesthetized with sodium pentobarbital $(50 \mathrm{mg} / \mathrm{kg}$ body weight, intraperitoneal) and transcardially perfused with PBS followed by 4\% PFA in $0.1 \mathrm{M}$ 
331 phosphate buffer ( $\mathrm{pH}$ 7.4). The brains were removed, postfixed in the same fixative overnight 332 at $4^{\circ} \mathrm{C}$, and immersed in $15 \%$ and $30 \%$ sucrose in PBS overnight for cryoprotection.

333 Free-floating sections $(40 \mu \mathrm{m})$ were cut using a sliding microtome (REM-710; Yamato Kohki 334 Industrial Co., Ltd., Japan) and incubated in PBS with $0.3 \%$ Triton X-100 and 1\% bovine 335 serum albumin (BSA) for $1 \mathrm{hr}$ at room temperature. Then, the sections were incubated for 12 $336 \mathrm{hr}$ at room temperature with primary guinea pig anti-GFP antibody $(1: 1,000$; Frontier 337 Institute) diluted in PBS with $0.3 \%$ Triton X-100, 2\% BSA. The sections were washed in 338 PBS and incubated for $2 \mathrm{hr}$ in Alexa Fluor 488-conjugaed species-specific secondary 339 antibodies (Thermo Fisher Scientific, Waltham, MA, USA) diluted 1:500 in PBS with 0.3\%

340 Triton X-100 and 2\% BSA. Fluorescent images of GFP were captured using a fluorescent 341 microscope (BZ-X710, KEYENCE, Osaka, Japan). Induction of iCre activity in the $\mathrm{Oxtr}^{3 \times \mathrm{HA-iCreERT2}}$ line by oral administration of TMX Oxtr ${ }^{3 \times H A-i C r E E R T 2}:: G t(R O S A) S o r^{A i 9}$ double heterozygous males at 4 weeks were fed with powdered diet supplemented with tamoxifen (TMX, Sigma-Aldrich T5648) at the concentration of $2 \mathrm{mg} / \mathrm{g}$ for 5 consecutive days by using feeding jars (Yoshinobu et al., 2021) $(n=5)$. For the control experiments, double heterozygous males at 4 weeks were fed with normal diet for 5 consecutive days $(n=3)$. After 5 more days, brains were sampled at 5 weeks and immunostained as described above. To analyze effects of chronic TMX treatment on Oxt and Oxtr expressions, 5-week-old homozygous Oxtr ${ }^{1 \times P A-t d T o m}$ males were fed with the same protocol $(n=3)$. Normal diet was fed to mice with the same genotype as the control $(n=3)$. Brains were sampled at 6 weeks for immunostaining.

\section{Results}

356 Seamless insertions of epitope tag and functional gene cassettes into the Oxtr locus via CRISPR/Cas9 genome editing

To insert the epitope tag or reporter/recombinase sequences in-frame and $5^{\prime}$ to the stop codon of the Oxtr gene $2^{\text {nd }}$ exon, we selected the guide RNA sequences depicted in Fig.1a. The targeting strategies were basically the same for four knock-in lines; one or three copies of epitope tag sequences were directly fused 3 ' to the Oxtr coding sequences. For bicistronic expression, we inserted T2A self-cleaving peptide sequences followed by functional gene cassettes (tdTomato reporter or Cre recombinase coding sequences). We used the T2A sequences which had been successfully utilized for knock-in mouse generations, to 
365

366

367

368

369

370

371

372

373

374

375

376

377

378

379

380

381

382

383

384

385

386

387

388

389

390

391

392

393

394

395

396

397

398

399

simultaneously express two separate proteins at nearly equimolar amounts from the same mRNA (Tang et al., 2009; Trichas et al., 2008).

We next employed the cloning-free CRISPR/Cas9 system in which chemically synthesized guide RNAs (crRNA and tracrRNA) were combined with recombinant Cas9 nuclease protein to induce highly efficient genome editing in mouse fertilized eggs (Aida et al., 2015; Inoue et al., 2021), and successfully generated one founder mouse for each knock-in design. We crossed these knock-in founders with C57BL/6J mice to obtain heterozygous F1 generations, which were sequence-verified to ensure that they correctly harbored the designed knock-in cassettes. We then intercrossed F1 heterozygous mice to obtain homozygous F2 generations. For histological, electrophysiological, and viral analyses, we prepared homozygous knock-in mice after F3 generations. Complete genomic DNA sequences and genotyping primer sequences for the four knock-in lines are supplied as dna files (Extended Data DNA sequence Files). Detailed genotyping PCR conditions are listed in Extended Data Figure 1-2.

\section{The epitope tagging strategy precisely visualized the endogenous Oxtr protein} expression profiles and ensured the reliability of the knock-in strategy.

As depicted in Fig.1b and 2a, we first designed a dual-purpose Oxtr ${ }^{1 \times P A-t d T o m}$ allele, in which one copy of PA-tag can monitor endogenous Oxtr protein localization and the portion of the tdTomato cassette serves as a bright fluorescent reporter. To evaluate PA-tag and tdTomato expression profiles in this line, we used homozygous male brains at 2 weeks (postnatal day 14; P14) because Oxtr expression levels in mouse brains have been reported to peak at around 2-3 weeks (Newmaster et al., 2020). In the figures, we mainly presented the section equivalent to Bregma $-1.82 \mathrm{~mm}$, as this level contains several well-known regions that have been reported to express Oxtr. Although tdTomato fluorescence could be detected without immunostaining as described later (Extended Data Fig.3-1a, 3-1b), we double-stained the sections with anti-tdTomato and anti-PA-tag antibodies to validate their colocalization. As a result, we observed abundant tdTomato reporter signals in the cortical region (Newmaster et al., 2020), hippocampal dentate gyrus (DG) and anterior CA2 (aCA2)/CA3 (Raam et al., 2017), paraventricular thalamus (PVT) (Newmaster et al., 2020; Watarai et al., 2020), central nucleus of amygdala (CeA) (Mitre et al., 2016; Yoshida et al., 2009), dorsal endopiriform nucleus (DEn) (Sharma et al., 2019), and posteromedial cortical amygdaloid area (PMCo) (Newmaster et al., 2020) (Fig.2d), where considerable level of Oxtr expression has already been reported. Consistently, we also detected the presence of endogenous Oxtr protein by the C-terminally fused PA-tag in the same regions (Fig.2e); however, the signal intensity was not 
400

401

402

403

404

405

406

407

408

409

410

411

412

413

414

415

416

417

418

419

420

421

422

423

424

425

426

427

428

429

430

431

432

433

434

strong enough for higher magnification, as described below. These matched results indicated that the epitope tagging and T2A-dependent bicistronic strategies we employed properly functioned as expected. We therefore applied the same design for the Oxtr ${ }^{1 \times P A-i C r e}$ allele (Fig $1 b)$.

In the magnified confocal image of the primary somatosensory cortex barrel field $(\mathrm{S} 1 \mathrm{BF})$ in the $O x t r^{1 \times P A-t d T o m}$ brain (Fig.2h), tdTomato and PA-tag signals showed significant colocalization. However, we noticed that the use of anti-PA-tag antibody resulted in high back-ground staining within brain tissue. To increase the signal-to-noise $(\mathrm{S} / \mathrm{N})$ ratio, we next generated the $O x{ }^{3 \times P A}$ allele in which three tandem copies of PA-tags were simply fused to the C-terminus of Oxtr (Fig. $1 \mathrm{~b}$ and 2b). Consequently, the PA-tag signals from this $3 \times \mathrm{PA}$ allele (Fig.2f) perfectly coincided with those from $1 \times \mathrm{PA}$ in the Oxtr ${ }^{1 \times P A-t d T o m}$ allele (Fig.2e), and its $\mathrm{S} / \mathrm{N}$ ratio was improved. On comparing the magnified confocal images of the $\mathrm{S} 1 \mathrm{BF}$, the PA-tag signals in the Oxtr ${ }^{3 \times P A}$ line (Fig.2i) were much clearer than those in the Oxtr ${ }_{1 \times P A-t d T o m}$ allele (Fig.2h), along with relatively lower background staining. In Fig.2i-2l, we focused on somatostatin (SST) expressing interneurons in layer IV, since Oxtr-expressing interneurons have been found in some brain areas to recruit local inhibitory circuits, which might be a major mode of oxytocinergic modulation in the brain (Froemke and Young, 2021), as described in the Discussion section below.

We further designed a dual-purpose Oxtr ${ }^{3 \times H A-i C r E R R T 2}$ allele (Fig.1b and 2c). We newly employed three copies of HA-tags for endogenous Oxtr protein visualization, because a good antibody with lower background staining in the brain tissue was commercially available. To induce spatiotemporally controlled Cre recombinase expressions in mouse brains, we fused a mutated ligand-binding domain of the human estrogen receptor (ERT2) (Indra et al., 1999) with iCre recombinase. The detailed results for iCre inductions are described later in Fig.5. As shown in Fig.2g, the HA-tag signals from this $3 \times$ HA allele nicely coincided with those from the $1 \times \mathrm{PA}$ and $3 \times \mathrm{PA}$ alleles (Fig.2e and 2f). On the magnified confocal image of $\mathrm{Oxtr}^{3 \times \text { HA-iCreERT2 }}$ brain shown in Fig.2k, we observed clear HA-tag signals, with relatively low background staining. The fact that three independently generated epitope-tagged lines resulted in exactly the same expression profiles confirms the reliability of our knock-in strategies.

\section{$\mathrm{Oxtr}^{3 \times P A}$ and $\mathrm{Oxtr}^{3 \times \mathrm{HA}-\mathrm{iCreERT2}}$ mouse lines enabled visualization of endogenous Oxtr} protein at the subcellular level

We next employed spinning-disk confocal microscopy technology to observe epitope tagged Oxtr-expressing neurons at super-resolution. First, we obtained the Z-stacked 
3-D image of a $3 \times$ PA-tagged neuron in the S1BF, which was identified as an SST-positive interneuron (Fig.2j and Fig.2 Movie 1). We successfully detected the distribution of PA-tagged endogenous Oxtr proteins on the plasma membrane, as expected as a member of GPCRs, whereas SST was localized in the cytoplasm. This is the first evidence showing that Oxtr proteins reside both on the cell body and on nerve fibers. The super-resolution image from a $3 \times$ HA-tagged neuron further replicated the above observation, supporting the reliability of this approach (Fig.21 and Fig.2 Movie 2).

\section{C-terminally fused epitope tags did not disrupt the Oxtr protein function in vivo.}

Although our experiments have confirmed the advantage of epitope tagging in visualizing endogenous protein localization, the concern remained that C-terminally fused tags may alter Oxtr protein conformation and/or stability, affecting its functions. Since Oxt plays essential roles in lactation, pups from Oxtr knock-out mothers $\left(\mathrm{Oxtr}^{-/}\right)$have been reported to die soon after birth, due to the mother's defects in breastfeeding (Hidema et al., 2016; Takayanagi et al., 2005) (Extended Data Fig.2-1a). Therefore, we confirmed that all the homozygous mothers of our four knock-in lines had successfully nursed their pups. As shown in Extended Data Fig.2-1, the pups' digestive tracts were filled with milk (Fig.2-1b, 2-1c) and the mother's mammary glands were well developed (Fig.2-1d), indicating that the peripheral Oxt signaling pathway was intact in the homozygous females. In addition, we performed electrophysiological recordings in tdTomato positive neurons from the homozygous Oxtr ${ }_{1 \times P A-t d T o m}$ male, to confirm their responsiveness to agonist administration (details are shown in Fig. 3 and described later), supporting that $1 \times$ PA tagged Oxtr proteins could sufficiently mediate Oxt signals in the brain. These results indicate that fusion of one or three copies of the PA-tag or HA-tag to the C-terminus of Oxtr did not affect receptor functions in vivo. It has already been reported that a much larger tdTomato protein fused $\mathrm{C}$-terminally to the $\mathrm{\kappa}$ opioid receptor, which is also a member of the GPCR family, did not disrupt neuronal circuitries in the knock-in mouse brain (Chen et al., 2020). Therefore, we did not expect that the relatively smaller epitope tags we had used in the present study would affect Oxtr functions. Consistent with this assumption, all the knock-in lines we generated could be maintained by crossing homozygous males with homozygous females (Extended Data Fig.2-1e, 2-1f), without the need for laborious genotyping procedures. This is a notable advantage of our new lines.

\section{The tdTomato reporter in the Oxtr $^{1 \times P A-t d T o m}$ line precisely labeled Oxtr-expressing} neurons. 

fluorescent protein suitable for live imaging (Shaner et al., 2004), we were able to detect tdTomato signals in our homozygous Oxtr ${ }^{1 \times P A-t d T o m}$ brains without immunostaining (Extended

473 Data Fig.3-1a, 3-1b), allowing us to perform patch-clamp recordings on these cells (Fig.3a,

474 3b). We hypothesized that tdTomato-positive neurons should correctly respond to an oxytocin receptor agonist, TGOT, if the tdTomato expressions were faithfully regulated parallel to endogenous Oxtr expressions.

A subset of both glutamatergic and GABAergic neurons in the PFC has been reported to express Oxtr, which modulate female sociosexual behavior (Nakajima et al., 2014) and social memory (Tan et al., 2019). We first investigated the distribution of tdTomato-positive neurons in the PFC using immunohistochemistry, particularly in the prelimbic cortex (PrL) of Oxtr ${ }^{1 \times P A-t d T o m}$ homozygous brain (Extended Data Fig.3-1). We found that tdTomato-positive neurons mainly resided in the layer II and V of the PrL (Extended Data Fig.3-1c, 3-1d). Consistent with a previous report (Tan et al., 2019), we identified the neurons in layer II by Cux1 expression as glutamatergic neurons (Extended Data Fig.3-1e, 3-1f), while the neurons in layer V were confirmed by SST expression as GABAergic interneurons (Extended Data Fig.3-1g, 3-1h).

To test whether these tdTomato-positive neurons in $O x{ }^{1 \times P A-t d T o m}$ mice faithfully 489 and perform in vitro electrophysiological analyses using a cell-attached patch-clamp 490 configuration (Fig.3a, 3b). We tested the neurons in layer II and V separately (Fig.3a), to test 491 the possibility that they might have distinct responsiveness to Oxt ligands to modulate synaptic sites or membrane potentials. As the results, in tdTomato-positive layer II neurons,

493 TGOT significantly increased the spontaneous firing rate from $2.7 \pm 1.0 \mathrm{~Hz}$ to $7.6 \pm 2.6 \mathrm{~Hz}(n$ $494=6 ; P=0.0312$, Wilcoxon test, Figure 3c, 3d). In tdTomato-positive layer V neurons, TGOT 495 also considerably increased the spontaneous firing rate from $0.99 \pm 0.7 \mathrm{~Hz}$ to $7.3 \pm 3.3 \mathrm{~Hz}(n$ $496=6 ; P=0.0312$, Wilcoxon test, Figure 3e, 3f). Additionally, we confirmed that these TGOT-induced increases in spontaneous firing rates in both layer II and V neurons were 498 inhibited by the presence of an Oxtr antagonist, OTA (Extended Data Fig.3-2a, 3-2b). These 499 results clearly demonstrate that tdTomato-positive cells in Oxtr ${ }^{1 \times P A-t d T o m}$ mice expresse 500 "functional" Oxtr and appropriately respond to its ligand, even in the presence of a 501 C-terminally fused PA-tag. Hence, tdTomato reporter signals in our Oxtr ${ }^{1 \times P A-t d T o m}$ line are a 502 reliable marker for Oxtr-expressing cells. 
504 The Oxtr $^{1 \times P A-i C r e}$ line sufficiently drove recombinase activities in the Oxtr-expressing

505 neurons.

506 To newly generate an effective Cre driver, we employed the 2A peptide-based

507 bicistronic expression system (Trichas et al., 2008) and codon-improved Cre recombinase

508 (iCre) sequences (Shimshek et al., 2002). For the evaluation of Cre activities in the brain, we

509 first crossed our Oxtr ${ }^{1 \times P A-i C r e}$ line with the well-known Cre reporter, Ai9 (Madisen et al.,

510 2010) to obtain double heterozygous mice. As depicted in Fig.4a, Cre-dependent stop signal

511 excision induces tdTomato expression from the Ai9 allele. We analyzed tdTomato expression 512 profiles in Oxtr $^{1 \times P A-i C r e /+}:: G t\left(R_{O S A}\right)$ Sor $^{A i 9 /+}$ male brains at P7, P14, and P21. We observed

513 tdTomato signals already at P7 (Fig.4b) and found that the reporter signals became more

514 abundant at P14 and P21 (Fig.4c, 4d). Once recombination occurs, the reporter gene remains

515 recombined thereafter, and consequently, the reporter is expressed even in the absence of

516 ongoing recombinase expressions. In other words, a significant proportion of tdTomato

517 expression in adult stages does not reflect real-time Cre activity but instead reflects a

518 cumulative effect of earlier events. Therefore, we concluded that the Ai9 reporter was

519 unsuitable for our purpose of evaluating Cre expressions in adult brains.

We next tried to immunostain Cre proteins in the brain tissue, using a commercially available anti-Cre recombinase antibody (clone 2D8, MAB3120, MERCK). This antibody has been reported to be successful in staining Cre proteins in transgenic lines carrying multiple copies of transgene (Mizuguchi et al., 2012). However, we could not detect Cre in the brain tissues of our iCre knock-in mouse line using this antibody.

To circumvent these issues, we employed AAV-PHP.eB vector harboring CBh-FLEX-GFP (kindly gifted by Dr. Hirokazu Hirai, Brain/MINDS project). AAV-PHP.eB has been developed to achieve efficient transport across the blood-brain barrier in adult mammalian brains for broad gene delivery (Chan et al., 2017). As depicted in Fig.4e, GFP expressions can be induced by Cre-dependent inversion. We therefore retro-orbitally injected AAV-PHP.eB vector into 6-week-old heterozygous males and analyzed their brains after 4 weeks. As the results, we observed abundant GFP signals in the cortical region, hippocampal DG and aCA2/CA3, PVT, CeA, DEn, and so forth, where Oxtr expression has previously been reported (Figure.4f-4j, Bregma $-1.82 \mathrm{~mm}$ ). Other section levels are shown in Extended Data Fig.4-1.

538

As we had designed the Oxtr ${ }^{1 \times P A-t d T o m}$ and $O x t r^{1 \times P A-i C r e}$ alleles based on the same concepts (Fig.1b and Fig.4e, 4k), their expression profiles should closely resemble each other. Predictably, we found that the tdTomato expression profiles from 11-week-old homozygous Oxtr ${ }^{1 \times P A-t d T o m}$ male brains (Fig.41-4p) were quite similar to those of iCre visualized by 
539 AAV-PHP.eB administration (Fig.4f-4j). Other section levels, shown in Extended Data

540 Fig.4-1, confirm the trend. These results ensured the reliability of our new Cre driver line.

541

542 A new genetic tool, Xxtr $^{3 \times H A-i C r e E R T 2}$ line, could be used to target Oxtr-expressing

543 neurons in a spatiotemporally controlled manner.

544 We next investigated whether the Oxtr ${ }^{3 \times H A-i C r e E R T 2}$ allele properly functioned as an 545 inducible Cre driver. To detect Cre activity in the brain, we crossed the Oxtr ${ }^{3 \times \text { HA-iCreERT2 }}$ line 546 with Ai9 to obtain double heterozygous mice. As depicted in Fig.5a, tamoxifen (TMX) binds 547 to iCre-ERT2 expressed in the cytoplasm, resulting in iCre translocation into the nucleus to 548 flip out the stop signal from Ai9 allele and elicit tdTomato expressions. We orally 549 administered TMX to 4-week-old males for 5 consecutive days by feeding them a 550 TMX-supplemented powdered diet (Fig.5b). For the control experiments, we fed 4-week-old males with normal diet for 5 consecutive days. After 5 more days, we sampled and analyzed their brains at the level of Bregma $-1.82 \mathrm{~mm}$. As shown in Fig. $5 \mathrm{c}$, the normal diet did not induce any leaky tdTomato reporter expression. Contrarily, the TMX-containing diet successfully induced iCre recombinase activity in the cortical region, hippocampal DG and aCA2/CA3, PVT, CeA, DEn, and PMCo, where Oxtr expression has previously been reported (Figure.5d-5i). In the constitutive iCre line, too many cells were labeled with tdTomato to be able to clearly distinguish each neuron (Fig.4d). However, reporter expression was only sparsely induced in the inducible iCre line, allowing the identification of cell morphology at single-cell resolution (Fig.5d-5i). As a result, we observed two types of Oxtr-expressing neurons with different morphologies and properties in the S1BF (Fig.5i). Consistent with the previous reports (Nakajima et al., 2014; Tan et al., 2019), some tdTomato-labelled neurons in layer II turned out to be Cux1-positive glutamatergic neurons (Fig.5j-51), while other tdTomato-labelled neurons in layer IV were identified as SST-positive interneurons (Fig.5m-5o).

These results demonstrated that the first-time inducible-Cre driver for controlled manner upon TMX administration.

Since subcutaneous implantations of a pellet containing estrogen into gonadectomized rodents have been reported to affect Oxt/Oxtr transcript levels (Bale and Dorsa, 1995; Garcia et al., 2017; Nomura et al., 2002; Patisaul et al., 2003; Vanya et al., 572 administrations of TMX, a selective estrogen receptor modulator, might change Oxt/Oxtr expression levels in the Oxtr ${ }^{3 \times \text { HA-iCreERT2 }}$ line. To analyze the effect of 5-days oral TMX 
574 treatment on Oxt/Oxtr expressions, we used homozygous Oxtr ${ }^{1 \times P A-t d T o m}$ mice, in which 575 real-time Oxtr expressions can be monitored as tdTomato signals, and examined 576 Oxt-expressing neurons by immunostaining (Extended Data Fig.5-1a). Although long-term 577 (12 days to 3 months) estrogen treatments have been reported to significantly increase Oxt 578 transcript levels in the paraventricular hypothalamic nucleus (PVN) (Nomura et al., 2002; 579 Patisaul et al., 2003) and supraoptic nucleus (SON) (Garcia et al., 2017), our relatively 580 short-term (5 days) TMX treatment did not affect Oxt expression levels in these nuclei 581 (Extended Data Fig5-1b). In addition, while long-term estrogen treatments have been 582 reported to affect Oxtr transcript levels in the bed nucleus of stria terminalis (BNST) (Garcia 583 et al., 2017), ventromedial hypothalamus (VMH) (Bale and Dorsa, 1995; Vanya et al., 1997), 584 and medial amygdala (MeA) (Garcia 2017), our 5-days TMX treatment had no effect on Oxtr 585 expression profiles in these brain areas (Extended Data Fig5-1c, 5-1d). Furthermore, while 586 radioactively labeled OTA binding to Oxtr has been reported to increase in the DEn and 587 PMCo by long-term estrogen treatment (Young et al., 1998), Oxtr expression levels itself 588 were not affected upon our TMX administrations (Extended Data Fig5-1d). From these 589 results, we concluded that 5-days TMX treatments have little effect on Oxt/Oxtr expression 590 profiles in young male mice. However, we need to carefully interpret the results obtained 591 from Oxtr ${ }^{3 \times H A-i C r e E R T 2}$ line by chronic TMX administrations because estrogens play key roles 592 in mediating social behaviors (Ervin et al., 2015; Laredo et al., 2014) as described in 593 Discussion section.

\section{Discussion}

Genetic tools that provide access to Oxtr-expressing cells remain limited. To

598 improve this situation, we have newly generated a series of genome-edited mouse lines that

599 are to make a valuable contribution to the growing research on oxytocin. We have created

600 simple and seamless knock-in designs that maximally preserve the endogenous

601 transcriptional regulation of the Oxtr gene.

602 The first achievement of the present study was the successful use of epitope tags to 603 visualize Oxtr protein distributions at the subcellular level for the first time. Two strategies, 604 the RNAscope-based in situ hybridization and the Oxtr-Venus knock-in mice, have previously 605 been utilized for Oxtr visualization. However, these methods detect transcript expression, but 606 not Oxtr protein localization per se. To overcome these limitations, we generated epitope tag 607 knock-in mice by genome editing. We employed the PA-tag, a 12 amino acid epitope derived 608 from human podoplanin, which could be detected using the rat monoclonal antibody, NZ-1 
609 (Fujii et al., 2014). This epitope-antibody combination has been reported to show a high

610 binding affinity of $K_{D}=4.9 \times 10^{-10}(M)$, which is much stronger than those of HA, FLAG, and

611 other affinity tag systems, and which has been successfully used to many biological

612 procedures in vitro (Fujii et al., 2014). In addition, we have previously reported the

613 applicability of the PA-tag system in mouse brains and testes in in vivo studies (Arimura et al.,

614 2020; Kimura et al., 2021). Here, we triplicated the PA-tag to improve the S/N ratio of

615 immunostaining, by which the background staining in brain tissues were reduced, allowing us

616 to successfully carry out super-resolution imaging of Oxtr protein. We also utilized the

617 HA-tag because it has been successfully employed for SLENDR, which enabled in vivo

618 protein labeling via genome editing in mammalian brains (Mikuni et al., 2016). Similarly,

619 utilizing three copies of the HA-tag enabled us to reconfirm the result from the $3 \times \mathrm{PA}$ tag

620 knock-in brains.

Although we have roughly demonstrated that Oxtr proteins are present not only on

622 cell bodies but also on neurites, it remains unclear on which part of neurites (axons, dendrites, 623 or both) they reside. To date, three types of oxytocinergic modulation on neurons have been

624 proposed (Froemke and Young, 2021). As the first-order modulation, Oxt directly depolarizes

625 excitatory cells that express Oxtr. In the second-order modulation, Oxt acts on inhibitory

626 interneurons expressing Oxtr, to regulate synaptic inhibitions. Furthermore, in the third-order

627 modulation, Oxtr is believed to reside on axon terminals, where the presynaptic Oxtr

628 activation enhances transmitter release and indirectly impacts on other excitatory

629 transmissions (Dölen et al., 2013; Nardou et al., 2019). However, the presence of Oxtr in

630 axon terminals has not yet been proven. Therefore, our in vivo super-resolution imaging

631 could serve as a significant step toward deciphering the subcellular distribution of Oxtr

632 protein in such studies.

633 The second achievement of the present study was the development of an Oxtr

634 reporter line expressing the bright red fluorescent protein, tdTomato, which is suitable for live

635 imaging. In vitro electrophysiological analyses were successfully carried out on

636 tdTomato-positive neurons in our reporter line. Such analyses may not have been possible in

637 the Oxtr-Venus line (Yoshida et al., 2009), because expression levels of Venus-positive cells

638 have been reported to largely decline after 3 weeks (Newmaster et al., 2020). In addition,

639 tdTomato's red fluorescence could be advantageous when calcium indicators such as the

640 genetically encoded GCaMPs are used, as they mainly contain GFP and emit green

641 fluorescence which overlaps with Venus signals.

642 Thirdly, we generated both constitutive and inducible Cre drivers for

643 Oxtr-expressing neurons. IRES elements, utilized in the existing two Oxtr-IRES-Cre knock-in 
644 lines, have been reported to occasionally cause lower expression of the downstream cistron

645 due to factors such as experimental cell types or cloned gene cassettes (Ibrahimi et al., 2009).

646 Accordingly, we employed the 2A peptide system, which allowed nearly equimolar

647 expression of two cistrons (Tang et al., 2009; Trichas et al., 2008). As we paid much attention

648 to maintain the endogenous Oxtr gene configuration in our knock-in allele, the design of our

649 Oxtr-T2A-iCre is indeed different from that of Oxtr-T2A-Cre-D, available from the Jackson

650 Laboratory (Daigle et al., 2018). While the latter inserted an exogenous bovine growth

651 hormone polyA signal and ignored the Oxtr's own endogenous polyA sequence (Extended

652 Data Fig.1-1e), our knock-in mice simply made use of the endogenous polyA signal instead

653 (Fig.1b). Unlike the systematically generated Cre allele as a part of a big project, our iCre

654 knock-in allele was carefully customized for the Oxtr locus, which is a promising

655 development for future oxytocin studies. In addition, our inducible Cre line was suitably

656 responsive to TMX administration. Although intraperitoneal injection is the most popular

657 TMX administration protocol, it may sometimes be toxic to cause the death of test animals.

658 Therefore, we employed an oral administration protocol (Andersson et al., 2010; Yoshinobu

659 et al., 2021) instead, to ensure the safe and sufficient induction of Cre activity.

660 TMX is a potent and clinically used selective estrogen receptor modulator that

661 blocks effects of estrogens in breast tissues but facilitates estrogen actions in others such as

662 uterus tissues (Osborne, 1998). Thus, chronic TMX treatment has the possibility to interfere

663 with estrogen function in multiple tissues including the brain. Estrogens are involved in

664 social behavior at multiple levels, from the detection and integration of social information to

665 more complex behavior such as social preference, aggression, and social learning and

666 memory (Ervin et al., 2015). As an example, in the hippocampus, estrogens are locally

667 synthesized and modulate memory-related synaptic plasticity by the rapid non-classical

668 indirect pathway via synapse-localized estrogen receptors (Laredo et al., 2014; Mukai et al.,

669 2010). We hence need to carefully consider the potential interference by TMX to the

670 behavioral outcomes from Oxtr manipulations in using Oxtr ${ }^{3 \times H A-i \text { CreERT2 }}$ mice.

671 Oxt and arginine vasopressin (Avp) are evolutionally related molecules that differ

672 only in two of the nine amino acids. Since their receptors also show significant structural

673 homology to one another, the selectivity of Oxt and Avp for their own receptor is not absolute.

674 Considerable receptor crosstalk has been reported to occur among Oxt, Avp and their

675 receptors (Carter, 2017; Song and Albers, 2018). While exogenously administrated Avp can

676 act on Oxtr in higher concentration and so can do Oxt on Avp receptor 1a (Avpr1a), limited

677 lines of evidence indicate that endogenously released Oxt and Avp can produce functionally

678 significant responses by activating each other's receptors (Song and Albers, 2018). Based on 
receptor autoradiography approach with relatively low resolution, Oxtr and Avprla appear to show distinct and non-overlapping expression in the rodent brain (Dumais and Veenema, 2016). However, it remains still unknown whether the localization of Oxtr and Avprla is mutually exclusive at the synaptic levels, and thus the possibility of crosstalk following synaptic release of these peptides should rigorously be examined. In this context, our new genetic tools could play a part in clarifying these receptors' distribution with much higher resolution, deepening our understanding of ligand-receptor interactions for Oxt and Avp. Generation of Avprla genome-edited mouse lines might further help precisely visualize its endogenous expression profiles.

Oxt is expected as a potential therapeutic for social deficits represented by autism spectrum disorders. Managing socio-emotional behavior in health and disease to increase resilience to chronic stress has become much more important, especially given the ongoing COVID-19 pandemic (Grinevich and Neumann, 2021). Although the preclinical trials of exogenous Oxt administration via intranasal delivery are currently in progress (Quintana et al., 2021), second-generation strategies to potentiate endogenous Oxt signaling should be preferred in the future (Ford and Young, 2021). Moreover, a fundamental shift from the traditional brain-wide chronic pharmacological intervention to a circuit-specific approach is now anticipated, owing to significant advances in our understanding of the neural circuit mechanisms underlying social behavior in animal models (Ford and Young, 2021). Hence, to establish dependable and effective Oxt-based therapeutic interventions, we need reliable animal models to help uncover the mechanisms underlying Oxt-Oxtr circuitry. Our new genetic tools would play a significant role in meeting this demand and we are going to make the four knock-in lines generated in this study quickly and widely available. This will eventually help deepen our knowledge for the elaborated molecular machinery and functional regions of Oxt-Oxtr actions in our own brain.

\section{Citations}

\section{Aida T, Chiyo K, Usami T, Ishikubo H, Imahashi R, Wada Y, Tanaka KF, Sakuma T,} Yamamoto T, Tanaka K (2015) Cloning-free CRISPR/Cas system facilitates functional cassette knock-in in mice. Genome Biol 16:87.

Andersson KB, Winer LH, Mørk HK, Molkentin JD, Jaisser F (2010) Tamoxifen administration routes and dosage for inducible Cre-mediated gene disruption in mouse hearts. Transgenic Res 19:715-725.

Arimura N, Okada M, Taya S, Dewa K, Tsuzuki A, Uetake H, Miyashita S, Hashizume K, 
Shimaoka K, Egusa S, Nishioka T, Yanagawa Y, Yamakawa K, Inoue YU, Inoue T, Kaibuchi K, Hoshino M (2020) DSCAM regulates delamination of neurons in the developing midbrain. Sci Adv 6:eaba1693.

Bale TL, Dorsa DM (1995) Sex differences in and effects of estrogen on oxytocin receptor messenger ribonucleic acid expression in the ventromedial hypothalamus. Endocrinology 136:27-32.

Brizzard B (2008) Epitope tagging. Biotechniques 44:693-695.

Carter CS (2017) The Oxytocin-Vasopressin Pathway in the Context of Love and Fear. Front Endocrinol (Lausanne) 8:356.

Chan KY, Jang MJ, Yoo BB, Greenbaum A, Ravi N, Wu W-L, Sánchez-Guardado L, Lois C, Mazmanian SK, Deverman BE, Gradinaru V (2017) Engineered AAVs for efficient noninvasive gene delivery to the central and peripheral nervous systems. Nat Neurosci 20:1172-1179.

Chen C, Willhouse AH, Huang P, Ko N, Wang Y, Xu B, Huang LHM, Kieffer B, Barbe MF, Liu-Chen LY (2020) Characterization of a knock-in mouse line expressing a fusion protein of $\mathrm{k}$ opioid receptor conjugated with tdtomato: 3-dimensional brain imaging via clarity. eNeuro 7:1-18.

Daigle TL et al. (2018) A Suite of Transgenic Driver and Reporter Mouse Lines with Enhanced Brain-Cell-Type Targeting and Functionality. Cell 174:465-480.e22.

Deisseroth K (2011) Optogenetics. Nat Methods 8:26-29.

Dölen G, Darvishzadeh A, Huang KW, Malenka RC (2013) Social reward requires coordinated activity of nucleus accumbens oxytocin and serotonin. Nature 501:179-184.

Dumais KM, Veenema AH (2016) Vasopressin and oxytocin receptor systems in the brain: Sex differences and sex-specific regulation of social behavior. Front Neuroendocrinol 40:1-23.

Ervin KSJ, Lymer JM, Matta R, Clipperton-Allen AE, Kavaliers M, Choleris E (2015) Estrogen involvement in social behavior in rodents: Rapid and long-term actions. Horm Behav 74:53-76.

Ford CL, Young LJ (2021) Translational opportunities for circuit-based social neuroscience: advancing 21 st century psychiatry. Curr Opin Neurobiol 68:1-8.

Froemke RC, Young LJ (2021) Oxytocin, Neural Plasticity, and Social Behavior. Annu Rev Neurosci 44:359-381.

Fujii Y, Kaneko M, Neyazaki M, Nogi T, Kato Y, Takagi J (2014) PA tag: A versatile protein tagging system using a super high affinity antibody against a dodecapeptide derived from human podoplanin. Protein Expr Purif 95:240-247. 
Garcia AN, Bezner K, Depena C, Yin W, Gore AC (2017) The effects of long-term estradiol treatment on social behavior and gene expression in adult female rats. Horm Behav $87: 145-154$.

Gerfen CR, Paletzki R, Heintz N (2013) GENSAT BAC Cre-Recombinase Driver Lines to Study the Functional Organization of Cerebral Cortical and Basal Ganglia Circuits. Neuron 80:1368-1383.

Grinevich V, Neumann ID (2021) Brain oxytocin: how puzzle stones from animal studies translate into psychiatry. Mol Psychiatry 26:265-279.

Grund T, Tang Y, Benusiglio D, Althammer F, Probst S, Oppenländer L, Neumann ID, Grinevich V (2019) Chemogenetic activation of oxytocin neurons: Temporal dynamics, hormonal release, and behavioral consequences. Psychoneuroendocrinology 106:77-84.

Hidema S, Fukuda T, Hiraoka Y, Mizukami H, Hayashi R, Otsuka A, Suzuki S, Miyazaki S, Nishimori K (2016) Generation of Oxtr cDNA ${ }^{H A}$-Ires-Cre Mice for Gene Expression in an Oxytocin Receptor Specific Manner. J Cell Biochem 117:1099-1111.

Horiai M, Otsuka A, Hidema S, Hiraoka Y, Hayashi R, Miyazaki S, Furuse T, Mizukami H, Teruyama R, Tamura M, Bito H, Maejima Y, Shimomura K, Nishimori K (2020) Targeting oxytocin receptor (Oxtr)-expressing neurons in the lateral septum to restore social novelty in autism spectrum disorder mouse models. Sci Rep 10:22173.

Hung LW, Neuner S, Polepalli JS, Beier KT, Wright M, Walsh JJ, Lewis EM, Luo L, Deisseroth K, Dölen G, Malenka RC (2017) Gating of social reward by oxytocin in the ventral tegmental area. Science (80- ) 357:1406-1411.

Ibrahimi A, Velde G Vande, Reumers V, Toelen J, Thiry I, Vandeputte C, Vets S, Deroose C, Bormans G, Baekelandt V, Debyser Z, Gijsbers R (2009) Highly efficient multicistronic lentiviral vectors with peptide $2 \mathrm{~A}$ sequences. Hum Gene Ther 20:845-860.

Indra AK, Warot X, Brocard J, Bornert J-M, Xiao J-H, Chambon P, Metzger D (1999) Temporally-controlled site-specific mutagenesis in the basal layer of the epidermis: comparison of the recombinase activity of the tamoxifen-inducible Cre-ERT and Cre-ERT2 recombinases. Nucleic Acids Res 27:4324-4327.

Inoue YU, Morimoto Y, Yamada M, Kaneko R, Shimaoka K, Oki S, Hotta M, Asami J, Koike E, Hori K, Hoshino M, Imayoshi I, Inoue T (2021) An Optimized Preparation Method for Long ssDNA Donors to Facilitate Quick Knock-In Mouse Generation. Cells 10:1076.

Jurek B, Neumann ID (2018) The oxytocin receptor: From intracellular signaling to behavior. Physiol Rev 98:1805-1908.

Kimura R, Inoue YU, Kikkawa T, Tatehana M, Morimoto Y, Inada H, Oki S, Inoue T, Osumi 
N (2021) Detection of REST expression in the testis using epitope-tag knock-in mice generated by genome editing. Dev Dyn. DOI: 10.1002/dvdy.417

King LB, Walum H, Inoue K, Eyrich NW, Young LJ (2016) Variation in the Oxytocin Receptor Gene Predicts Brain Region-Specific Expression and Social Attachment. Biol Psychiatry 80:160-169.

Laredo SA, Villalon Landeros R, Trainor BC (2014) Rapid effects of estrogens on behavior: Environmental modulation and molecular mechanisms. Front Neuroendocrinol 35:447458.

Madisen L, Zwingman TA, Sunkin SM, Oh SW, Zariwala HA, Gu H, Ng LL, Palmiter RD, Hawrylycz MJ, Jones AR, Lein ES, Zeng H (2010) A robust and high-throughput Cre reporting and characterization system for the whole mouse brain. Nat Neurosci 13:133140.

Maldonado PP, Nuno-Perez A, Kirchner JH, Hammock E, Gjorgjieva J, Lohmann C (2021) Oxytocin Shapes Spontaneous Activity Patterns in the Developing Visual Cortex by Activating Somatostatin Interneurons. Curr Biol 31:322-333.e5.

Marlin BJ, Mitre M, D'amour JA, Chao M V., Froemke RC (2015) Oxytocin enables maternal behaviour by balancing cortical inhibition. Nature 520:499-504.

Marsh N, Marsh AA, Lee MR, Hurlemann R (2020) Oxytocin and the Neurobiology of Prosocial Behavior. The Neuroscientist 107385842096011.

Menon R, Grund T, Zoicas I, Althammer F, Fiedler D, Biermeier V, Bosch OJ, Hiraoka Y, Nishimori K, Eliava M, Grinevich V, Neumann ID (2018) Oxytocin Signaling in the Lateral Septum Prevents Social Fear during Lactation. Curr Biol 28:1066-1078.e6.

Mikuni T, Nishiyama J, Sun Y, Kamasawa N, Yasuda R (2016) High-Throughput, High-Resolution Mapping of Protein Localization in Mammalian Brain by In Vivo Genome Editing. Cell 165:1803-1817.

Mitre M, Marlin BJ, Schiavo JK, Morina E, Norden SE, Hackett TA, Aoki CJ, Chao M V., Froemke RC (2016) A Distributed Network for Social Cognition Enriched for Oxytocin Receptors. J Neurosci 36:2517-2535.

Miwa H, Fukaya M, Watabe AM, Watanabe M, Manabe T (2008) Functional contributions of synaptically localized NR2B subunits of the NMDA receptor to synaptic transmission and long-term potentiation in the adult mouse CNS. J Physiol 586:2539-2550.

Mizuguchi R, Naritsuka H, Mori K, Yoshihara Y (2012) Tbr2 Deficiency in Mitral and Tufted Cells Disrupts Excitatory-Inhibitory Balance of Neural Circuitry in the Mouse Olfactory Bulb. J Neurosci 32:8831-8844.

Mukai H, Kimoto T, Hojo Y, Kawato S, Murakami G, Higo S, Hatanaka Y, Ogiue-Ikeda M 
819 (2010) Modulation of synaptic plasticity by brain estrogen in the hippocampus. Biochim Biophys Acta - Gen Subj 1800:1030-1044.

Nagy A, Gertsenstein M, Vintersten K, Behringer R (2003) Manipulating the Mouse Embryo: A Laboratory Manual, 3rd ed. New York: Cold Spring Harvor Laboratory Press.

Nakajima M, Görlich A, Heintz N (2014) Oxytocin Modulates Female Sociosexual Behavior through a Specific Class of Prefrontal Cortical Interneurons. Cell 159:295-305.

Nardou R, Lewis EM, Rothhaas R, Xu R, Yang A, Boyden E, Dölen G (2019) Oxytocin-dependent reopening of a social reward learning critical period with MDMA. Nature 569:116-120.

Neumann ID, Slattery DA (2016) Oxytocin in General Anxiety and Social Fear: A Translational Approach. Biol Psychiatry 79:213-221.

Newmaster KT, Nolan ZT, Chon U, Vanselow DJ, Weit AR, Tabbaa M, Hidema S, Nishimori K, Hammock EAD, Kim Y (2020) Quantitative cellular-resolution map of the oxytocin receptor in postnatally developing mouse brains. Nat Commun 11:1885.

Nomura M, McKenna E, Korach KS, Pfaff DW, Ogawa S (2002) Estrogen receptor- $\beta$ regulates transcript levels for oxytocin and arginine vasopressin in the hypothalamic paraventricular nucleus of male mice. Mol Brain Res 109:84-94.

Onaka T, Takayanagi Y (2019) Role of oxytocin in the control of stress and food intake. J Neuroendocrinol 31:e12700.

Osborne CK (1998) Tamoxifen in the Treatment of Breast Cancer. N Engl J Med 339:16091618.

Owen SF, Tuncdemir SN, Bader PL, Tirko NN, Fishell G, Tsien RW (2013) Oxytocin enhances hippocampal spike transmission by modulating fast-spiking interneurons. Nature 500:458-462.

Patisaul HB, Scordalakes EM, Young LJ, Rissman EF (2003) Oxytocin, But Not Oxytocin Receptor, is Regulated by Oestrogen Receptor $\beta$ in the Female Mouse Hypothalamus. J Neuroendocrinol 15:787-793.

Quintana DS, Lischke A, Grace S, Scheele D, Ma Y, Becker B (2021) Advances in the field of intranasal oxytocin research: lessons learned and future directions for clinical research. Mol Psychiatry 26:80-91.

Raam T, McAvoy KM, Besnard A, Veenema A, Sahay A (2017) Hippocampal oxytocin receptors are necessary for discrimination of social stimuli. Nat Commun 8:1-14.

Resendez SL, Namboodiri VMK, Otis JM, Eckman LEH, Rodriguez-Romaguera J, Ung RL, Basiri ML, Kosyk O, Rossi MA, Dichter GS, Stuber GD (2020) Social Stimuli Induce Activation of Oxytocin Neurons Within the Paraventricular Nucleus of the 
Hypothalamus to Promote Social Behavior in Male Mice. J Neurosci 40:2282-2295.

Rothman JS, Silver RA (2018) Neuromatic: An integrated open-source software toolkit for acquisition, analysis and simulation of electrophysiological data. Front Neuroinform 12:1-21.

Ryan PJ, Ross SI, Campos CA, Derkach VA, Palmiter RD (2017)

Oxytocin-receptor-expressing neurons in the parabrachial nucleus regulate fluid intake. Nat Neurosci 20:1722-1733.

Shaner NC, Campbell RE, Steinbach PA, Giepmans BNG, Palmer AE, Tsien RY (2004) Improved monomeric red, orange and yellow fluorescent proteins derived from Discosoma sp. red fluorescent protein. Nat Biotechnol 22:1567-1572.

Sharma K, LeBlanc R, Haque M, Nishimori K, Reid MM, Teruyama R (2019) Sexually dimorphic oxytocin receptor-expressing neurons in the preoptic area of the mouse brain. PLoS One 14:e0219784.

Shimshek DR, Kim J, Hübner MR, Spergel DJ, Buchholz F, Casanova E, Stewart AF, Seeburg PH, Sprengel R (2002) Codon-improved Cre recombinase (iCre) expression in the mouse. Genesis 32:19-26.

Song Z, Albers HE (2018) Cross-talk among oxytocin and arginine-vasopressin receptors: Relevance for basic and clinical studies of the brain and periphery. Front Neuroendocrinol 51:14-24.

Takayanagi Y, Yoshida M, Bielsky IF, Ross HE, Kawamata M, Onaka T, Yanagisawa T, Kimura T, Matzuk MM, Young LJ, Nishimori K (2005) Pervasive social deficits, but normal parturition, in oxytocin receptor-deficient mice. Proc Natl Acad Sci 102:1609616101.

Tan Y, Singhal SM, Harden SW, Cahill KM, Nguyen DTM, Colon-Perez LM, Sahagian TJ, Thinschmidt JS, De Kloet AD, Febo M, Frazier CJ, Krause EG (2019) Oxytocin receptors are expressed by glutamatergic prefrontal cortical neurons that selectively modulate social recognition. J Neurosci 39:3249-3263.

Tang W, Ehrlich I, Wolff SBE, Michalski A-M, Wölfl S, Hasan MT, Lüthi A, Sprengel R (2009) Faithful Expression of Multiple Proteins via 2A-Peptide Self-Processing: A Versatile and Reliable Method for Manipulating Brain Circuits. J Neurosci 29:86218629.

Trichas G, Begbie J, Srinivas S (2008) Use of the viral 2A peptide for bicistronic expression in transgenic mice. BMC Biol 6:1-13.

Vanya QJ, Jenab S, Ogawa S, Adan RAM, Burbach PH, Pfaff DW (1997) Effects of Estrogen on Oxytocin Receptor Messenger Ribonucleic Acid Expression in the Uterus, 
Pituitary, and Forebrain of the Female Rat. Neuroendocrinology 65:9-17.

Watarai A, Tsutaki S, Nishimori K, Okuyama T, Mogi K, Kikusui T (2020) The blockade of oxytocin receptors in the paraventricular thalamus reduces maternal crouching behavior over pups in lactating mice. Neurosci Lett 720:134761.

Yoshida M, Takayanagi Y, Inoue K, Kimura T, Young LJ, Onaka T, Nishimori K (2009) Evidence that oxytocin exerts anxiolytic effects via oxytocin receptor expressed in serotonergic neurons in mice. J Neurosci 29:2259-2271.

Yoshinobu K, Araki Masatake, Morita A, Araki Miyuki, Kokuba S, Nakagata N, Araki K (2021) Tamoxifen feeding method is suitable for efficient conditionalknockout. Exp Anim 70:91.

Young LJ, Wang Z, Donaldson R, Rissman EF (1998) Estrogen receptor $\alpha$ is essential for induction of oxytocin receptor by estrogen. Neuroreport 9:933-936.

\section{Figure legends}

Figure 1. A series of Oxtr knock-in mouse lines generated by CRISPR/Cas9 genome editing

a, Targeting strategy to insert a functional cassette just upstream from the translational stop codon (TGA) of mouse Oxtr gene locus is schematically outlined. Oxtr gene has two coding regions shown as solid black bars that are divided by a long intron. Its 3' UTR shown as an open bar is also relatively long. PAM and guide sequences to recruit CRISPR components onto the Oxtr stop codon are depicted at the top. Solid grey bar indicates a knock-in cassette. Note that the endogenous Oxtr gene configuration including the long intron and 3' UTR are intactly preserved in the resulting knock-in allele even after the genome editing. For comparison with already-existing knock-in lines, see Extended Data Fig.1-1.

b, Four knock-in alleles generated in this study are schematically depicted. Epitope tag sequences $(1 \times \mathrm{PA}, 3 \times \mathrm{PA}$, or $3 \times \mathrm{HA})$ are fused $3^{\prime}$ to the Oxtr coding sequences. T2A self-cleaving peptide sequences are employed for bicistronic functional cassette expression (tdTomato, iCre, and ERT2) in Oxtr-expressing cells. Complete genomic DNA sequences and genotyping primer sequences for four knock-in lines are supplied as .dna files (Extended Data DNA sequence Files 1-1, 1-2, 1-3, and 1-4). Detailed genotyping PCR conditions are listed in Extended Data Figure 1-2. 
923 Extended Data Figure 1-1. Already-existing reporter/Cre lines for Oxtr

924 visualization/manipuration

925 a. Endogenous Oxtr gene configuration is schematically depicted. Oxtr gene has two coding

926 regions shown as solid black bars that are divided by a long intron (11.5 kb). Its 3' UTR

927 shown as an open bar is also relatively long $(3.3 \mathrm{~kb})$.

928 b. Oxtr-Venus knock-in allele (Yoshida et al., 2009). As the 1st coding exon is replaced with

929 Venus sequences followed by bovine growth hormone (GH) polyA signal, this allele lacks the

930 endogenous configuration of intron and 3'UTR sequences.

931 c. Oxtr cDNA $A^{H A}$-IRES-Cre knock-in allele (Hidema et al., 2016). As is the case with

932 Oxtr-Venus in b, the 1st coding exon is replaced with Oxtr CDNA-HA-IRES-Cre cassette.

933 Although the C-terminally fused HA-tag is detected in uterus tissues, it cannot be detected in 934 brain tissues.

935 d. Oxtr ${ }^{\text {Cre:GFP }}$ knock-in allele (Ryan et al., 2017). While the long intron is preserved, the

936 exogenously integrated polyA signal is prioritized over the endogenous Oxtr's polyA.

937 Exogenous polyA signals might affect stabilities of mRNAs.

938 e. Oxtr-T2A-Cre-D knock-in allele (Daigle et al., 2018). As is the case in d, the exogenously

939 introduced polyA signal is prioritized over the endogenous Oxtr's polyA.

940 f. Oxtr-Cre BAC transgenic allele (Gerfen et al., 2013). Although BAC transgenesis is

941 undoubtedly a useful technique, a non-native genomic context via random integration might

942 unexpectedly modify endogenous expression profiles.

943

944 Extended Data DNA sequence Files (supplied as a zip file)

945 Complete genomic DNA sequences and genotyping primer sequences for four knock-in lines

946 generated in this study are supplied as .dna files that can be opened by using SnapGene

947 software.

948

949 Extended Data Figure 1-2. Genotyping primers and PCR conditions used in this study

950 a. Names of four knock-in lines generated in this study are listed.

951 b. Genotyping primer names are listed.

952 c. Primer sequences (5' to 3') are listed.

953 d. PCR enzymes used for genotyping are listed.

954 e. PCR products' size for knock-in alleles and wild type allele are listed.

955 f. PCR conditions for genotyping are listed.

956 g. Electrophoresis conditions are listed.

957 
959 Figure 2. Epitope-tagging strategy precisely visualizes the endogenous Oxtr protein 960 expression profiles and ensures the reliability of knock-in strategies.

961 a-c, Schematic diagram of Oxtr ${ }^{1 \times P A-t d T o m}$ allele, Oxtr ${ }^{3 \times P A}$ allele, and $O x t r^{3 \times H A-i C r E E R T 2}$ allele are

962 arranged on the top. Resulting Oxtr-expressing neurons are depicted below them. To visualize

963 endogenous Oxtr protein localization, PA-tag or HA-tag are fused to the C-terminus of

964 receptor protein and detected by anti-tag antibodies. To enhance the detection, three copies of

965 PA-tag or HA-tag are tandemly connected. C-terminally fused PA-tag/HA-tag do not disrupt

966 the receptor function in vivo as shown in Extended Data Fig.2-1. tdTomato reporter and

967 iCre-ERT2 recombinase are bicistronically expressed in the cytoplasm by employing the T2A

968 self-cleaving peptide system.

969 d,e, Expression profiles of tdTomato reporter and 1×PA-tag in the Oxtr ${ }^{1 \times P A-t d T o m}$ homozygous

970 brain from 2-week-old male are arranged (equivalent to Bregma $-1.82 \mathrm{~mm}$ ). tdTomato

971 expressions are observed in the areas that have been reported to express Oxtr, such as the

972 primary somatosensory cortex barrel field (S1BF), hippocampal dentate gyrus (DG) and

973 anterior CA2 (aCA2)/CA3, paraventricular thalamus (PVT), central nucleus of amygdala

974 (CeA), dorsal endopiriform nucleus (DEn), and posteromedial cortical amygdaloid area

975 (PMCo). Notably, PA-tag signals are also observed in the same regions, indicating the

976 T2A-dependent bicistronic expression system works well.

977 f, Expression profiles of $3 \times \mathrm{PA}$-tag in the $\mathrm{Oxtr}^{3 \times P A}$ homozygous brain from 2-week-old male

978 nicely match up with those of tdTomato in $\mathbf{d}$ and $1 \times \mathrm{PA}$-tag in $\mathbf{e}$.

979 g, Expression profiles of $3 \times$ HA-tag in the Oxtr ${ }^{3 \times H A-i C r e E R T 2}$ homozygous brain from

980 2-week-old male well coincide with those of $1 \times$ PA-tag in $\mathbf{e}$ and $3 \times$ PA-tag in $\mathbf{f}$. Note that

981 independently generated three epitope-tagged lines result in the same expression profiles,

982 ensuring the reliability of knock-in strategies.

983 h. Magnified confocal image of the boxed area (S1BF, layer IV) in d-e indicates tdTomato

984 signals nicely coincide with PA-tag staining. However, this line is not suitable for higher

985 magnification due to its unwanted back-ground staining of PA-tag antibody.

986 i. Enlarged confocal image of the boxed area (S1BF, layer IV) in $\mathbf{f}$ shows clearer signals

987 derived from $3 \times$ PA-tag than those from $1 \times$ PA-tag in $\mathbf{h}$.

988 j. Super resolution image of a somatostatin (SST)-positive PA-tagged Oxtr-expressing neuron 989 indicates PA-tag signals are observed both on the surface of cell body and on the neurites,

990 whereas SST is localized in the cytoplasm. 3-D movie of this interneuron is supplied as Fig.2

991 Movie 1. 
992 k. Magnified confocal image of the boxed area (S1BF, layer IV) in $\mathbf{g}$ shows much clearer 993 signals derived from $3 \times$ HA-tag than those from $1 \times$ PA-tag in $\mathbf{h}$.

994 I. Super resolution image of an SST-positive HA-tagged Oxtr-expressing neuron indicates

995 HA-tag signals are observed on the surface of cell body and on the neurites, reconfirming the

996 result from 3xPA-tag knock-in line in j. 3-D movie of this interneuron is supplied as Fig.2

997 Movie 2

998

999

1000

Movie 1. Super resolution image of an SST-positive PA-tagged Oxtr-expressing neuron

PA-tag signals shown in green are observed both on the surface of cell body and on the neurites, whereas SST signals shown in magenta are localized in the cytoplasm. DAPI nuclear staining signals are shown in blue.

1003

Movie 2. Super resolution image of an SST-positive HA-tagged Oxtr-expressing neuron HA-tag signals shown in magenta are observed both on the surface of cell body and on the neurites, whereas SST signals shown in green are localized in the cytoplasm. DAPI nuclear staining signals are shown in blue.

1008

1009

1010

\section{Extended Data Figure 2-1. Homozygous knock-in males and females do not have any} problems in their reproductive functions.

1011

a. Pups from Oxtr knock-out mothers $\left(\mathrm{Oxtr}^{--}\right)$has been reported to die soon after birth due to

1012 mother's defects in breast-feeding since Oxt plays essential roles in lactation (Hidema et al., 2016). Pups' digestive tracts are not filled with milk.

1014

b. Digestive tract of a pup from homozygous $O x t r{ }^{1 \times P A-t d T o m}$ mother is filled with milk,

1015 indicating homozygous mothers have no problems in lactation.

1016

c. Digestive tract of a pup from homozygous $O x t{ }^{1 \times P A-i C r e}$ mother is filled with milk, indicating the peripheral Oxt signaling pathway is intact.

1018

d. Mammary glands of homozygous Oxtr ${ }^{1 \times P A-t d T o m}$ mother are well-developed.

1019

e. A homozygous $O x t r^{1 \times P A-i C r e}$ mother can nurse her pups by lactating.

1020

f. A pair of homozygous $O x t r^{1 \times P A-i C r e}$ mother and father, and their homozygous progenies.

1021

Homozygous knock-in males are fertile.

1022

1023

1024

Figure 3. Functional verification of tdTomato-positive neurons in $\mathrm{Oxtr}^{1 \times P A-t d T o m}$ mice by 1025 
1026 a, Schematic diagram of a mouse brain coronal section which shows placement of recording

1027 electrodes in layer II and layer V of the prelimbic cortex (PrL). IL; infralimbic cortex.

1028 Distribution of tdTomato-positive neurons in the PrL of Oxtr ${ }^{1 \times P A-t d T o m}$ homozygous brain are 1029 shown in Extended Data Fig.3-1.

1030 b, Representative differential interference contrast (DIC; left), fluorescent (red; middle), and 1031 merged (right) images of a layer V neuron in the PrL from 4-week-old Oxtr ${ }^{1 \times \text { PA-tdTom }}$ 1032 homozygous male are arranged.

$1033 \mathbf{c}$, Representative cell-attached recording traces from a layer II tdTomato-positive cell in the 1034 PrL. Left panel shows the baseline firing rate. Upon treatment of oxytocin receptor agonist, 1035 TGOT, the firing rates are significantly increased as shown in right panel. Scale bar, $10 \mathrm{pA}, 1$ 1036 sec.

1037 d, Quantification of spontaneous firing activities from layer II tdTomato-positive cells in the $1038 \operatorname{PrL}(n=6$ cells from 6 mice). Light red lines represent individual neurons' responses. Red 1039 circles with line represent average response. Presence of $1 \mu \mathrm{M}$ TGOT significantly increases 1040 the firing rate. ${ }^{*} P<0.05$ by Wilcoxon's test. As shown in Extended Data Fig.3-2a, this 1041 TGOT-induced increase in firing rates is inhibited by presence of Oxtr antagonist, OTA.

1042 e, Representative cell-attached recording traces from a layer V tdTomato-positive cell in the 1043 PrL. Left panel indicates the baseline firing rate. On treatment of TGOT, the firing rates are 1044 considerably increased as shown in right panel. Scale bar, $50 \mathrm{pA}, 1 \mathrm{sec}$.

1045 d, Quantification of firing activities from layer V tdTomato-positive cells in the $\operatorname{PrL}(n=6$ 1046 cells from 6 mice). Administration of $1 \mu \mathrm{M}$ TGOT considerably increases the spontaneous 1047 firing rate. ${ }^{*} P<0.05$ by Wilcoxon's test. As shown in Extended Data Fig.3-2b, this 1048 TGOT-induced increase in firing rates is inhibited by presence of OTA.

1049

\section{Extended Data Figure 3-1. Characteristics of tdTomato-positive neurons in the} prelimbic cortex (PrL)

1052 Serial coronal sections of 5-week-old homozygous Oxtr ${ }^{1 \times P A-t d T o m}$ male brain at Bregma 1.94 $1053 \mathrm{~mm}$ are arranged.

1054 a, Endogenous tdTomato fluorescence can be detected without immunostaining.

$1055 \mathbf{b}$, Enlarged image of the boxed area in $\mathbf{a}$.

1056 c, Enhanced tdTomato fluorescent signals are detected with anti-tdTomato antibody.

1057 d, Enlarged image of the boxed area in $\mathbf{c}$ indicates tdTomato-positive cells reside in both 1058 layer II/III and in layer V of the PrL.

1059 e, f, tdTomato-expressing neurons in layer II express Cux1, one of the glutamatergic neuron 1060 markers. 

interneuron markers.

\section{Extended Data Figure 3-2. Blocking effects of oxytocin receptor antagonist, OTA on} TGOT-induced increase of spontaneous firing activities in tdTomato-positive neurons

a, Mean spontaneous firing activities of layer II tdTomato-positive cells in the prelimbic cortex (PrL) from Oxtr ${ }^{1 \times \text { PA-tdTom }}$ homozygous males. Light red lines represent individual neurons' responses. Red circles with line represent average response. Firing activities remain unchanged in the presence of $1 \mu \mathrm{M}$ OTA (left panel, $n=6$ cells from 4 mice). $1 \mu \mathrm{M}$ TGOT application in the presence of $1 \mu \mathrm{M}$ OTA do not increase firing activities of tdTomato-positive cells (right panel, $n=3$ cells from 3 mice). ns, not significant. b, Mean spontaneous firing activities of layer V tdTomato-positive cells in the PrL from Oxtr ${ }^{1 \times P A-t d T o m}$ homozygous males are arranged. Firing activities remain unchanged the in presence of $1 \mu \mathrm{M}$ OTA (left panel, $n=7$ cells from 6 mice). $1 \mu \mathrm{M}$ TGOT application in the presence of $1 \mu \mathrm{M}$ OTA do not affect firing rates of tdTomato-positive cells (right panel, $n=5$ cells from 3 mice)

Figure 4. Xxtr $^{1 \times P A-i C r e}$ mice reliably drive recombinase activities in Oxtr-expressing cells. a, Schematics of $O x t r{ }^{1 \times P A-i C r e}$ allele and Gt(ROSA)Sor ${ }^{A i 9}$ allele are depicted. b-d, tdTomato expression profiles in $\mathrm{Oxtr}^{1 \times P A-i C r e /+}:: G t(\operatorname{ROSA}) \operatorname{Sor}^{A i 9 /+}$ male brains at P7, P14, and P21 are arranged (equivalent to Bregma $-1.82 \mathrm{~mm}$ ). Constitutive tdTomato expressions from the $G t(R O S A)$ Sor locus after Cre-dependent stop signal excision gradually accumulate over time. tdTomato signals are observed already at P7 and then become more abundant at $\mathrm{P} 14$ and P21. GFP expression can be induced by Cre-dependent inversion.

1088 f, Expression profiles of GFP in the Oxtr ${ }^{1 \times P A-i C r e}$ heterozygous brain (Bregma $-1.82 \mathrm{~mm}$ ) from 1089 11-week-old male to which AAV-PHP.eB vector has been retro-orbitally injected at 6 weeks.

1090 Other section levels are shown in Extended Data Fig.4-1.

$1091 \mathbf{g}-\mathbf{j}$, Enlarged images of the boxed areas in $\mathbf{f}$ are arranged. GFP expressions are observed in 1092 the areas that have been reported to express Oxtr, such as the S1BF, hippocampal DG and 1093 aCA2/CA3, PVT, CeA, and DEn, indicating this mouse line sufficiently drives Cre activities 1094 in Oxtr-expressing cells.

1095 k. Schematic diagram of $O x$ tr $^{1 \times P A-t d T o m}$ allele is depicted. 
1096 I. tdTomato expression profiles in the Oxtr ${ }^{1 \times P A-t d T o m}$ homozygous brain (Bregma $-1.82 \mathrm{~mm}$ )

1097 from 11-week-old male are arranged. Other section levels are shown in Extended Data

1098 Fig.4-1.

$1099 \mathbf{m}-\mathbf{p}$, Enlarged images of the boxed areas in $\mathbf{I}$ are arranged. These tdTomato expression

1100 profiles correspond to the GFP expressions shown in $\mathbf{g}-\mathbf{j}$ induced by iCre upon AAV-PHPeB

1101 administration.

\section{Extended Data Figure 4-1. Atlas of iCre/tdTomato expression profiles}

1104 Left side; Expression profiles of GFP in Oxtr ${ }^{1 \times P A-i C r e}$ heterozygous brain from 11-week-old 1105 male to which AAV-PHP.eB CBh_FLEX-GFP vector has been retro-orbitally injected at 6 1106 weeks. Right side; tdTomato expression profiles in Oxtr ${ }^{1 \times P A-t d T o m}$ homozygous brain from 1107 11-week-old male.

1108 a,b, Bregma $1.78 \mathrm{~mm}$. M1; primary motor cortex, S1; primary somatosensory cortex, PrL;

1109 prelimbic cortex, DEn; dorsal endopiriform claustrum, IEn; intermediate endopiriform

1110 claustrum.

1111 c,d, Bregma $1.42 \mathrm{~mm}$. Cg; cingulate cortex, S1; primary somatosensory cortex, AcbC;

1112 accumbens nucleus, core, AcbSh; accumbens nucleus, shell.

1113 e,f, Bregma $0.62 \mathrm{~mm}$.

1114 g,h, Bregma -0.10 mm. LS; lateral septum.

$1115 \mathbf{i}, \mathbf{j}$, Bregma -0.58 mm. DEn; dorsal endopiriform nucleus.

1116 k, l, Bregma -2.30 mm. V2M; secondary visual cortex medial area, V1; primary visual cortex, 1117 V2L; secondary visual cortex lateral area, Au; auditory cortex, PMCo; posteromedial cortical 1118 amygdaloid area.

$1119 \mathbf{m}, \mathbf{n}$, Bregma $-2.92 \mathrm{~mm}$.

Figure 5. Cre recombinase activities can be reliably induced in Oxtr-expressing neurons upon tamoxifen administrations to the $\mathrm{Oxtr}^{3 \times H A-i C r e E R T 2}$ line.

1125 (TMX) binds to iCre-ERT2 expressed in the cytoplasm, resulting in iCre translocation into

1126 the nucleus to flip out stop signal from Ai9 allele and induce tdTomato expression.

b. Schematic diagram of experimental protocol. Oxtr ${ }^{3 \times H A-i C r e E R T 2}:: G t(R O S A)$ Sor $^{A i 9}$ double

1128 heterozygous mice can freely access to TMX-supplemented diet from Day 0 to Day 5. At Day

1129 10, brains are sampled and immunostained. For control experiments, TMX-free normal diet is 
1130 fed to same genotype mice. As shown in Extended Data Fig5-1, 5-days TMX treatment has

1131 no effects on Oxt/Oxtr expression profiles in 5-week-old males.

1132 c. TMX-free normal diet does not induce any leaky tdTomato signals in the brain from

1133 5-week-old male (Bregma -1.82 mm).

1134 d. TMX-supplemented diet induces tdTomato expressions in the cortical region, hippocampal

1135 DG and aCA2/CA3, PVT, CeA, DEn, and PMCo, where Oxtr expressions has been reported.

1136 The density of the tdTomato positive cells is much sparser than that induced by the

1137 constitutive iCre line shown in Fig 4d.

1138 e-h, Enlarged images of the boxed areas in $\mathbf{d}$ are arranged.

1139 i. Enlarged image of the S1BF in $\mathbf{d}$.

$1140 \mathbf{j}-\mathbf{l}$, Confocal image of the boxed area in $\mathbf{i}$, labeled with Cux1 immunostaining, reveals the

1141 morphology of Oxtr-expressing glutamatergic neuron in layer II. White arrowhead in $\mathbf{k}$

1142 indicates the colocalization of Cux 1 and tdTomato.

$1143 \mathbf{m - o}$, Confocal images of the boxed area in i, labeled with Cux1 and somatostatin (SST)

1144 immunostaining, reveals the morphologies of Oxtr-expressing SST-positive interneurons in

1145 layer IV. White arrows in $\mathbf{n}$ indicate the colocalization of SST and tdTomato.

1146

1147 Extended Data Figure 5-1. 5-days TMX treatment has no effect on Oxt/Oxtr

1148 expression profiles in male Oxtr $^{1 \times P A-t d T o m / 1 \times P A-t d T o m}$ mice.

1149 a. Schematic diagram of experimental protocol. 5-week-old homozygous Oxtr ${ }^{1 \times P A-t d T o m}$ males

1150 can freely access to TMX-supplemented diet from Day 0 to Day $5(n=3)$. At Day 10, brains

1151 are sampled for immunostaining. For control experiments, TMX-free normal diet is fed to

1152 mice with the same genotype $(\mathrm{n}=3)$.

1153 b. Oxt expression profiles in respective $O x t r^{1 \times P A-t d T o m / 1 \times P A-t d T o m}$ male brain are arranged

1154 (Bregma -0.94 mm). Left column shows Oxt-expressing neurons in the paraventricular

1155 hypothalamic nucleus (PVN) and supraoptic nucleus (SON) from normal diet conditions. In

1156 right column, 5-days TMX treatment has no effect on Oxt expression profiles in these nuclei.

1157 c. tdTomato expression profiles in respective $O x t r^{1 \times P A-t d T o m / 1 \times P A-t d T o m}$ male brain are arranged

1158 (Bregma $0.62 \mathrm{~mm}$ ). In this knock-in line, tdTomato monitors real-time Oxtr expressions. Left

1159 column shows tdTomato-expressing cells in the bed nucleus of stria terminalis (BNST) from

1160 normal diet conditions. In right column, 5-days TMX treatment has no effect on tdTomato

1161 expressions in the BNST.

1162 d. tdTomato expression profiles in respective $O x t r^{1 \times P A-t d T o m / 1 \times P A-t d T o m}$ male brain are arranged

1163 (Bregma -1.82 mm). Left column shows tdTomato-expressing cells in the ventromedial

1164 hypothalamus (VMH), medial amygdala (MeA), PMCo, and DEn from normal diet 
1165 conditions. In right column, 5-days TMX treatment has no effect on tdTomato expressions in 1166 these brain areas.

1167

1168 Materials Availability

1169 Four knock-in mouse lines generated in this study will be made available with a materials

1170 transfer agreement. 


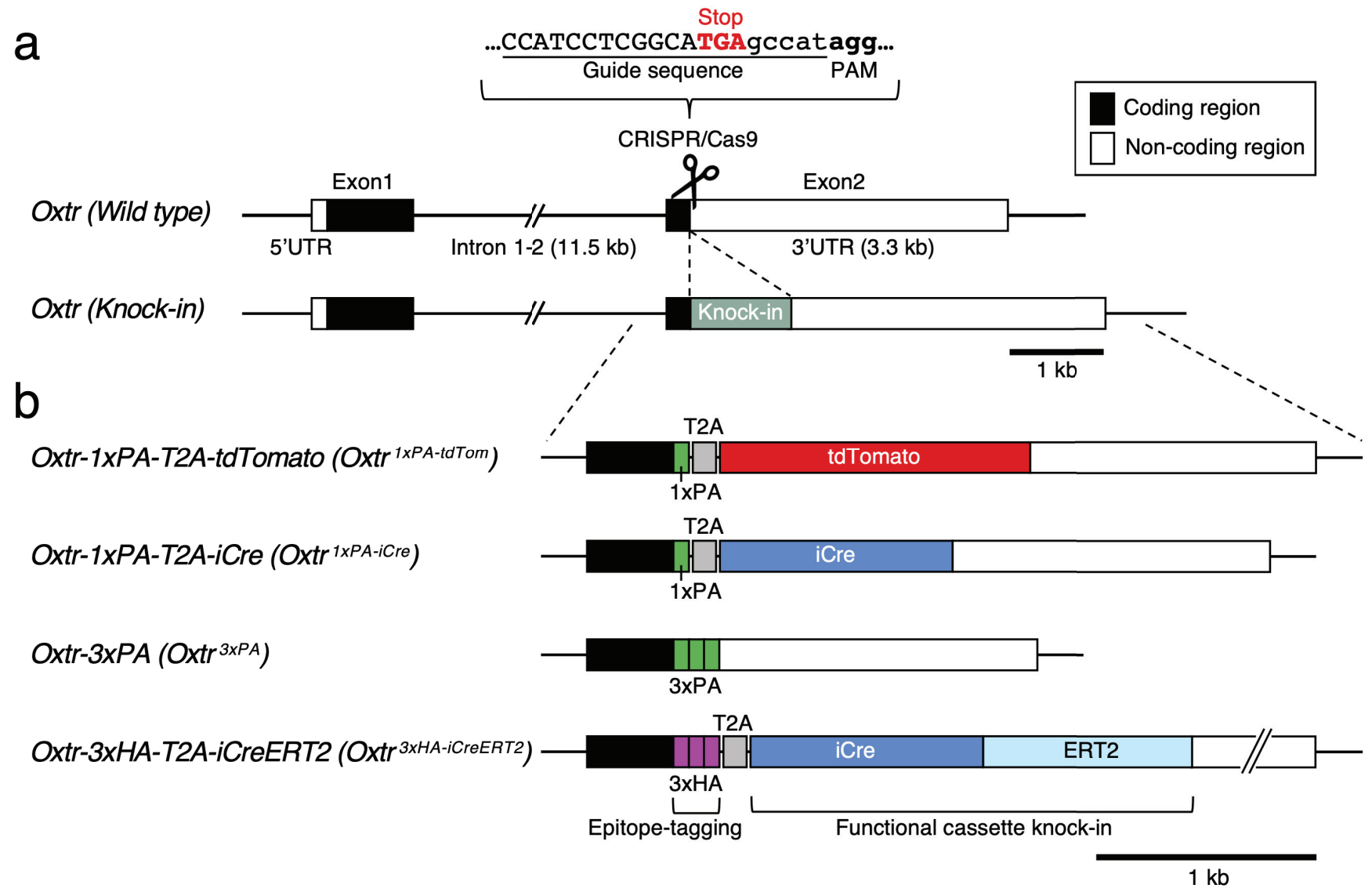


a
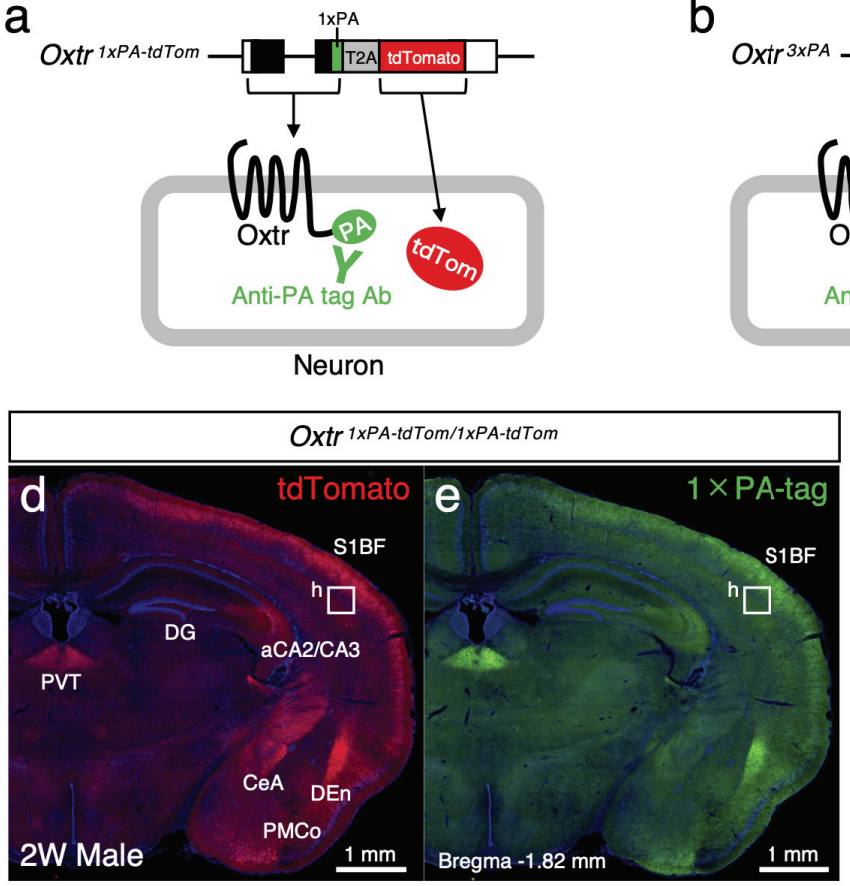

tdTomato DAPI b

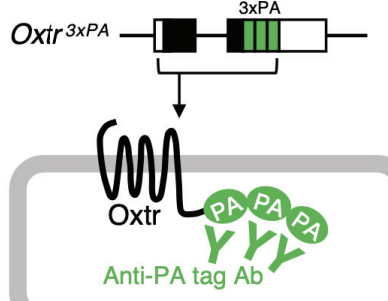

Neuron

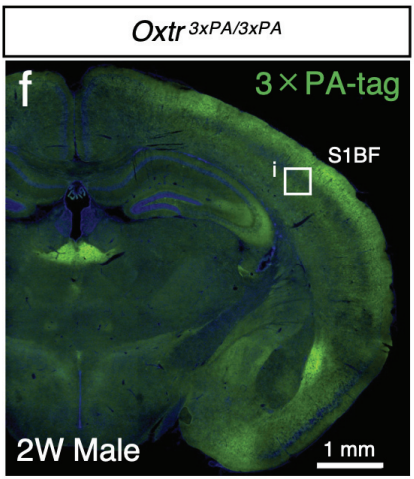

PA DAPI

C OXtr $3 \times$ HA-ICrEERT2

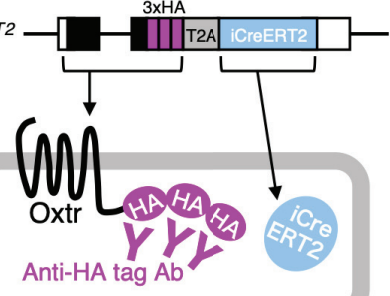

Neuron

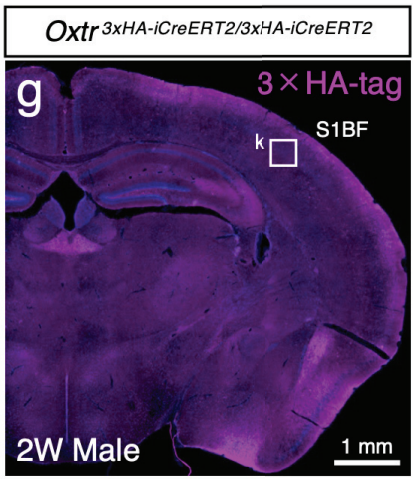

HA DAPI
Oxtr 1xPA-tdTom/1xPA-tdTom

Confocal

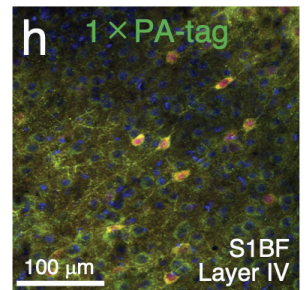

PA tdTomato DAPI
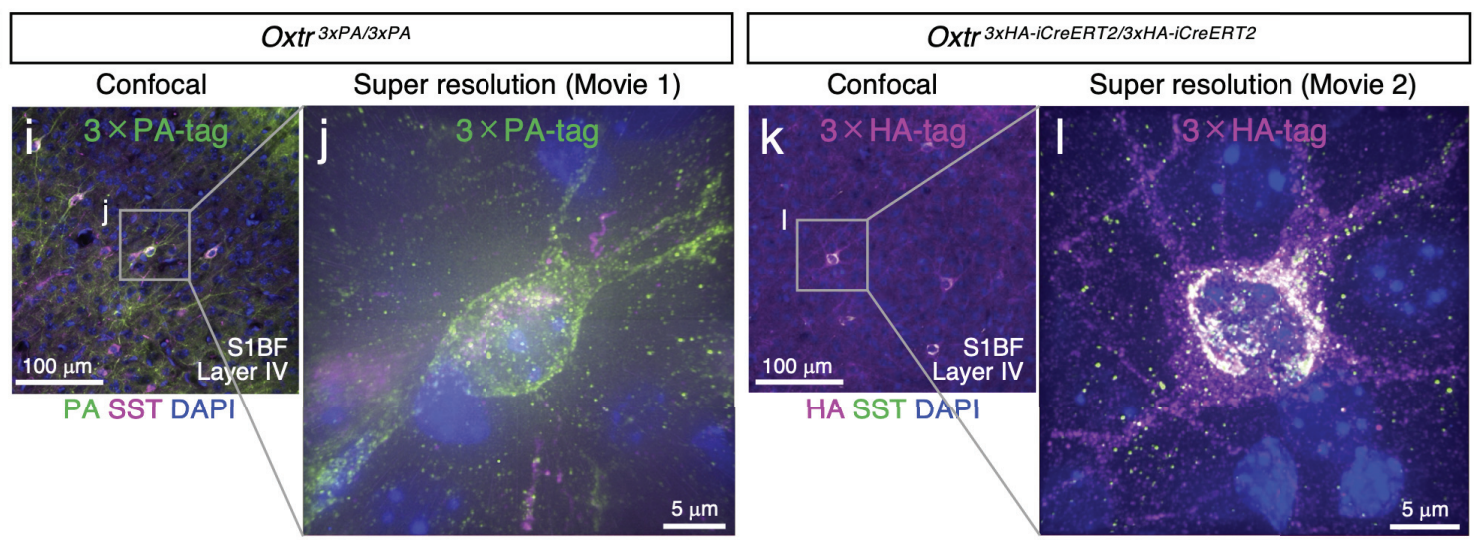

PA SST DAPI

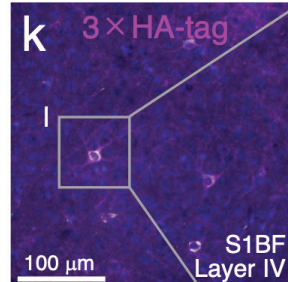

HA SST DAPI

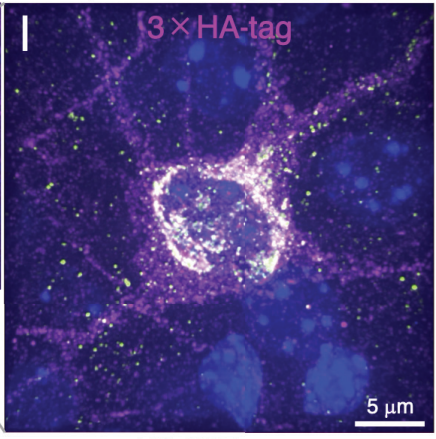

HA SST DAPI 
a

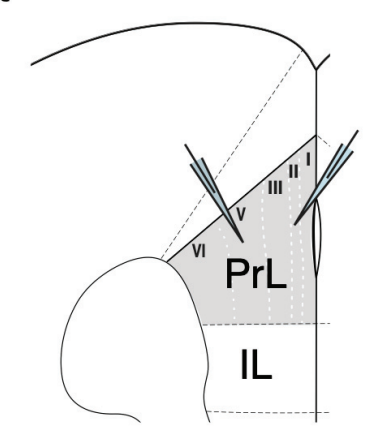

C

\section{Layer II}

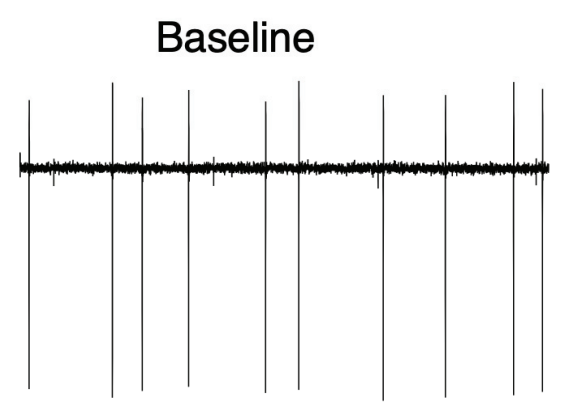

(d)

(U)

(u)

$<$

(2)

(3)

(d)

b

Layer V
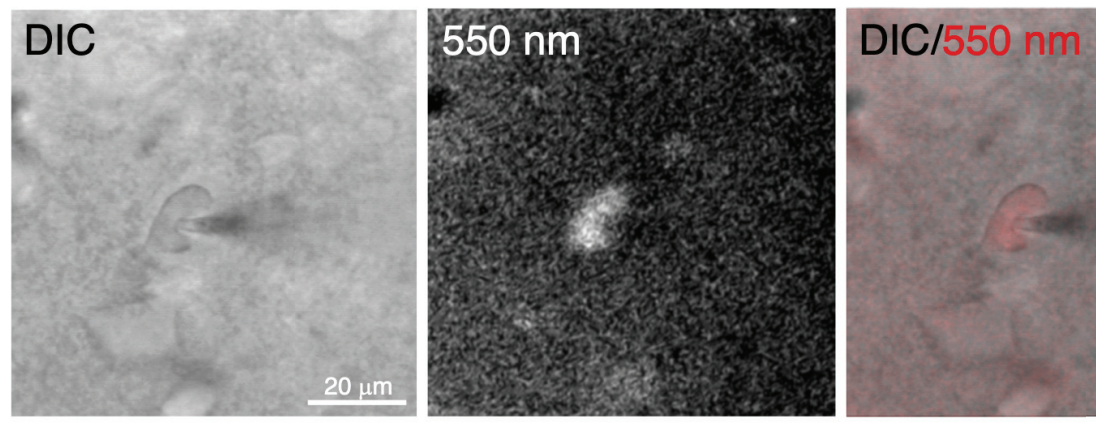

d
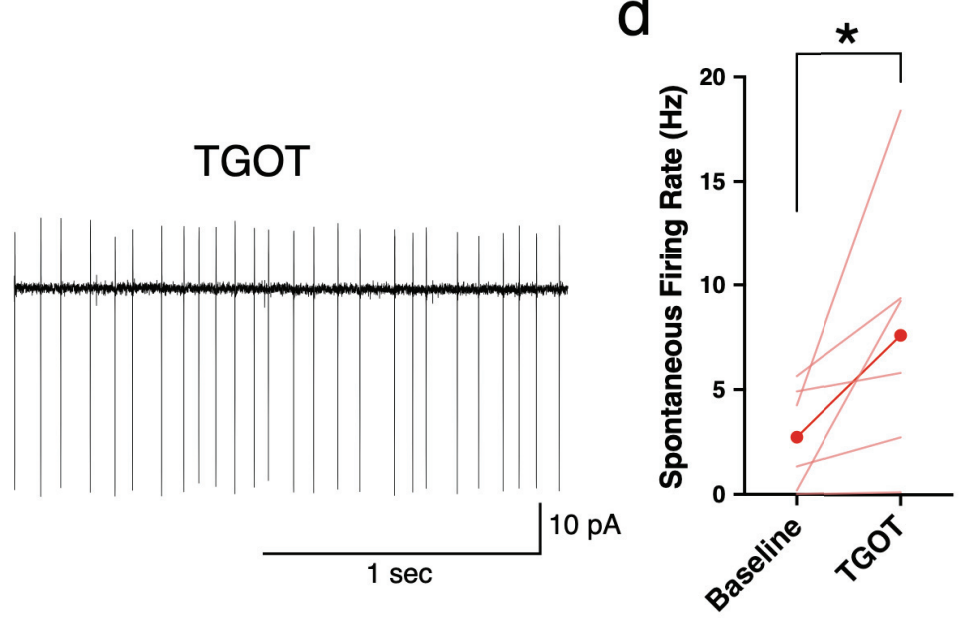

TGOT

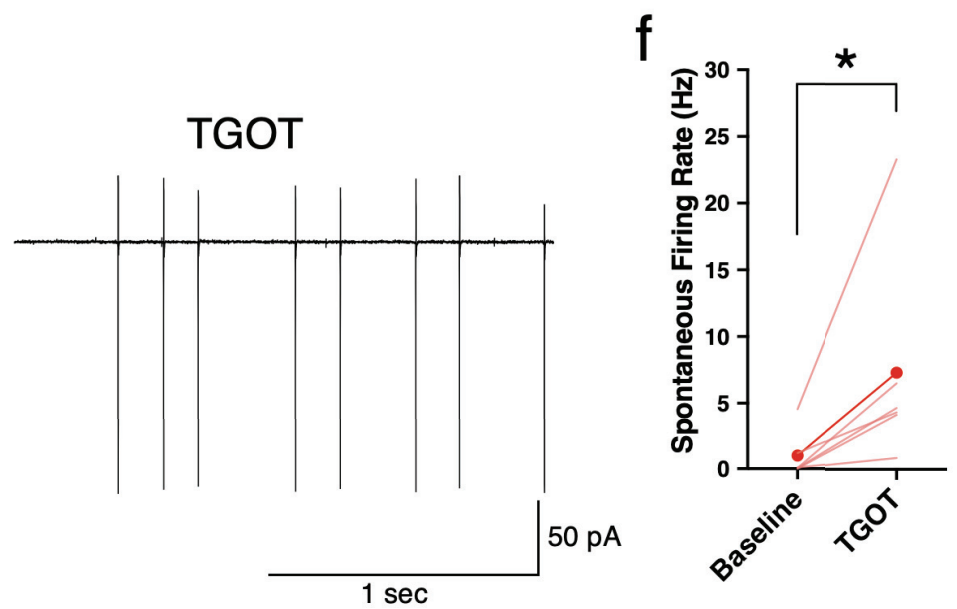


a

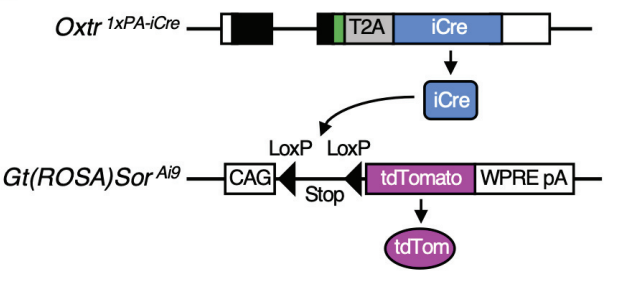

e

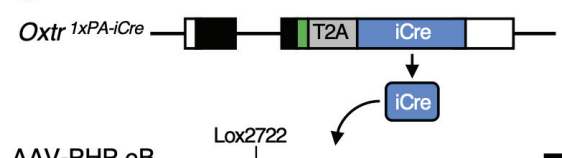

AAV-PHP.eB
CBh-FLEX-GFP CBh CBh-FLEX-GFP LOXP

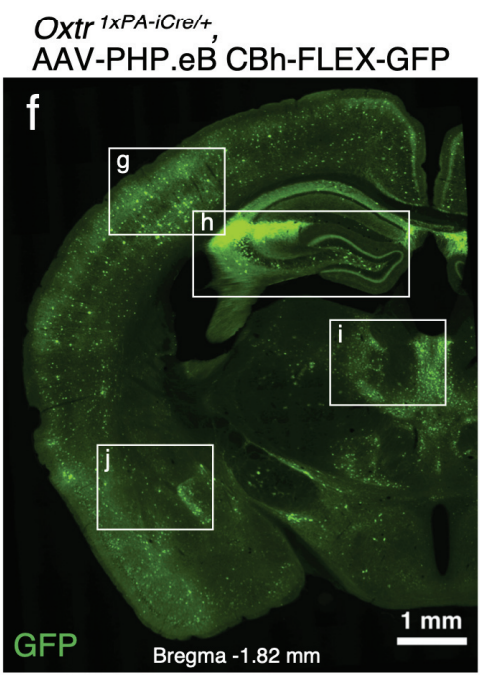

Male 6W, PHP.eB >11W Sampling
Oxtr ${ }^{i C r e /+}$ :: Gt(ROSA)26Sor ${ }^{\text {Aig/+ }}$ (Bregma -1.82 mm)

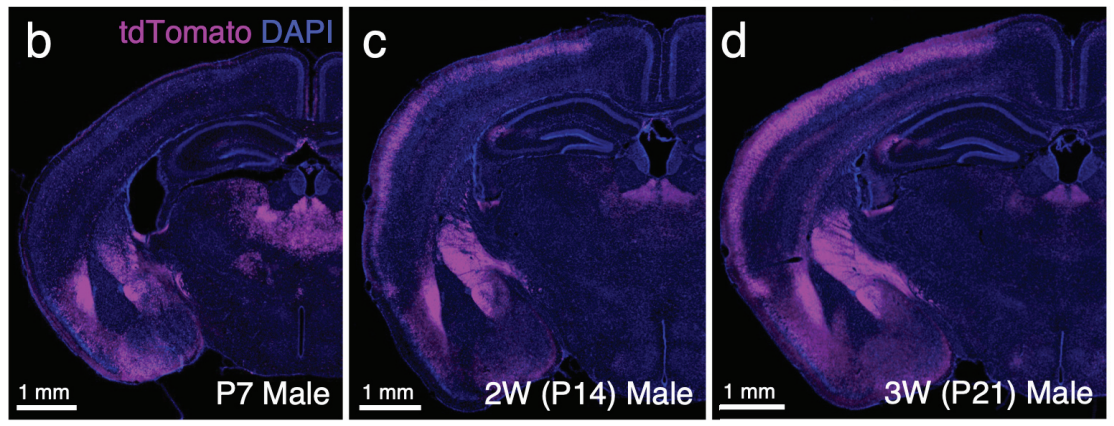

$\mathrm{k}$

Oxtr ${ }^{1 \times P A}$-tdTom $-\longrightarrow$ T2A

m s1BF tolTomato
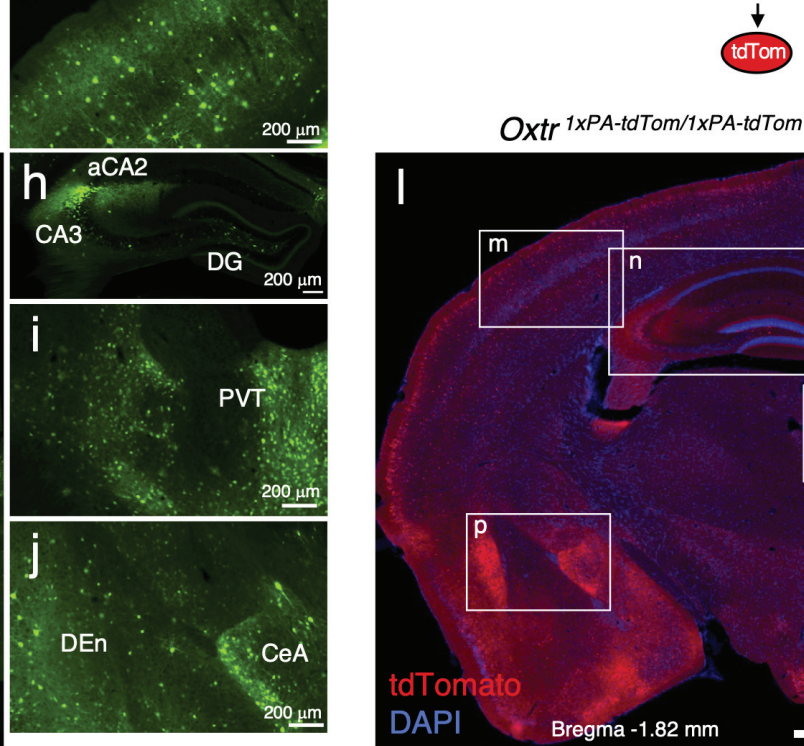

$200 \mu \mathrm{m}$
Oxtr 1xPA-tdTom/1xPA-tdTom

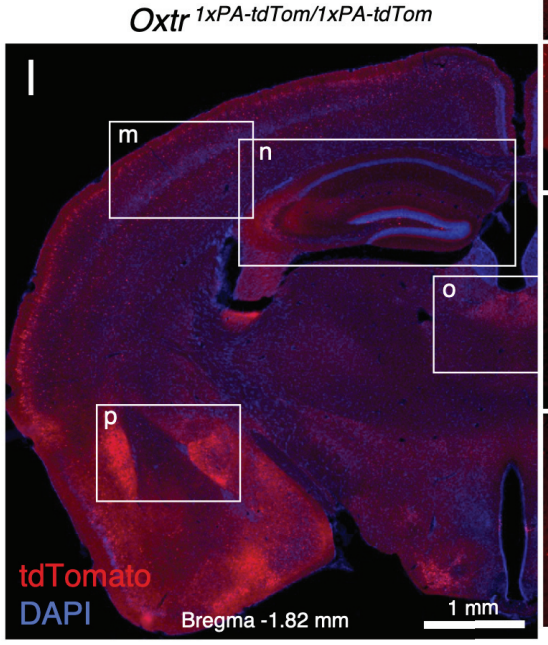

Male $11 \mathrm{~W}$

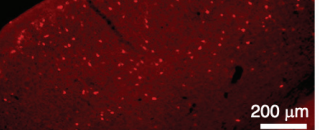

n aCA2

0

PVT
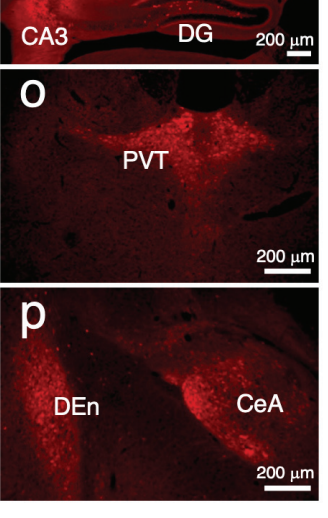

$200 \mu \mathrm{m}$ 

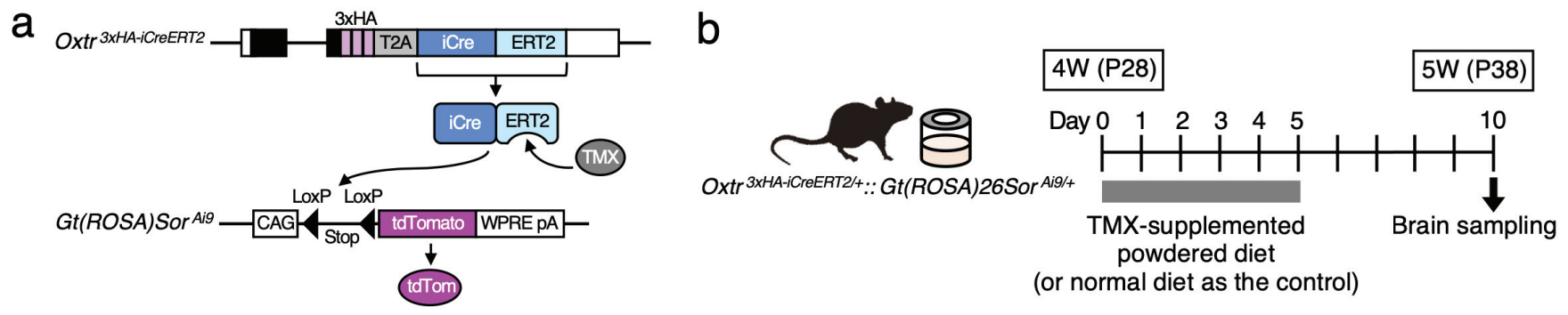

Oxtr 3xHA-iCreERT2/+:: Gt(ROSA)26Sor Aig/+
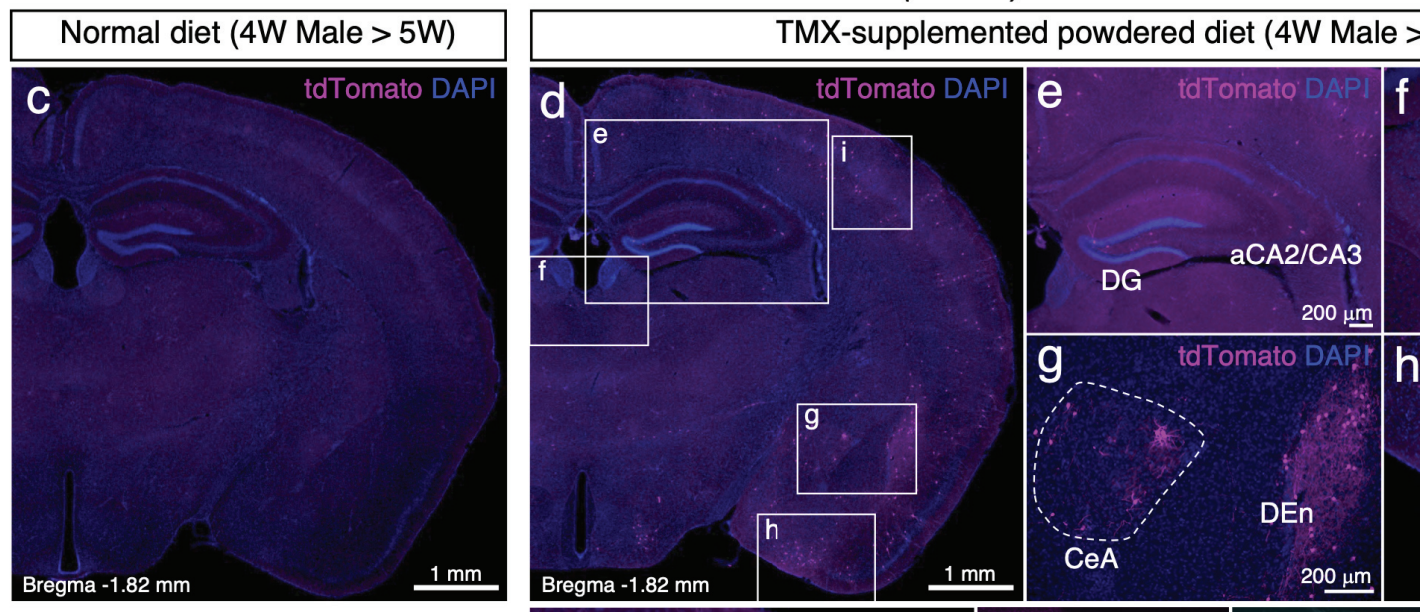

$>5 \mathrm{~W})$

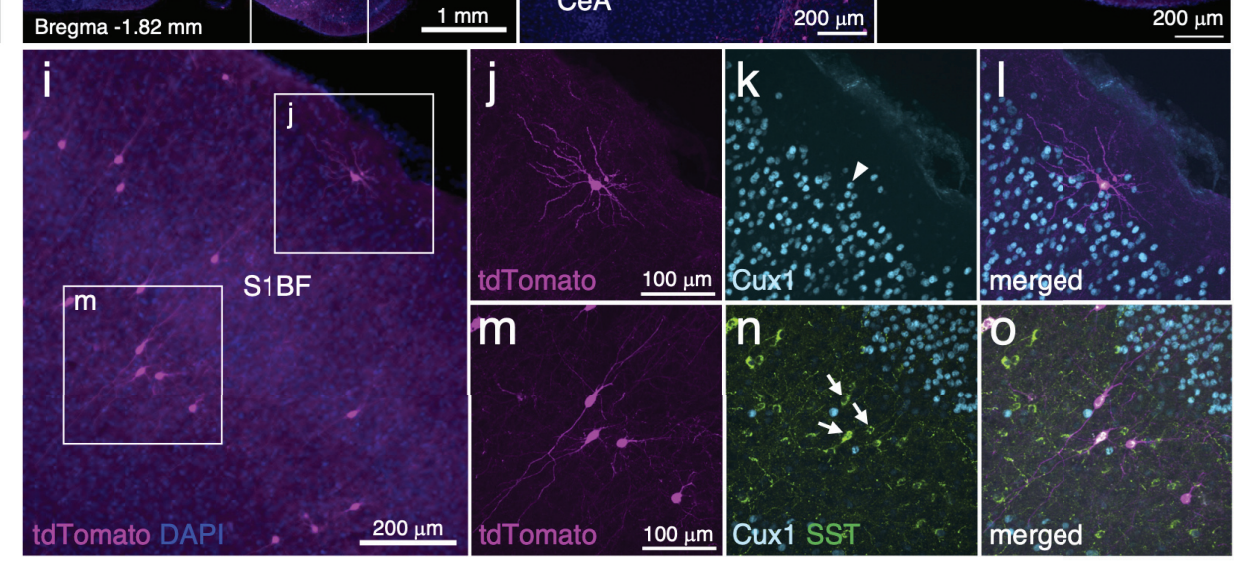

